\title{
Pd-catalyzed amination of dibromobiphenyls in the synthesis of macrocycles comprising two biphenyl and two polyamine moieties
}

\author{
Alexei N. Uglov, ${ }^{a}$ Alexei D. Averin, ${ }^{a}$ Alexei K. Buryak, ${ }^{b}$ and Irina P. Beletskaya ${ }^{a, b *}$ \\ ${ }^{a}$ Lomonosov Moscow State University, Department of Chemistry, Leninskie Gory 1-3, Moscow \\ 119 991, Russia \\ ${ }^{b}$ A.N. Frumkin Institute of Physical Chemistry and Electrochemistry, 31 Leninskii Prosp., \\ Moscow 119 991, Russia \\ E-mail: beletska@org.chem.msu.ru
}

Dedicated to Prof. Usein M. Dzhemilev on the occasion of his $65^{\text {th }}$ anniversary

\begin{abstract}
Two approaches to the synthesis of polyazamacrocycles containing two biphenyl and two polyamine moieties are described. The first route comprises the synthesis of $N, N^{\prime}-$ bis(bromobiphenyl) substituted di- and poly-amines by the reactions of 2.2-3 equivalents of 4,4'or 3,3'-dibromobiphenyls with corresponding di- and polyamines followed by the reaction with a second molecule of the amine. The second approach includes the synthesis of bis(polyamine) substituted biphenyls by the reactions of 4,4'- or 3,3'-dibromobiphenyls with four equivalents of di- and poly-amines with subsequent reaction of these in situ prepared intermediates with dibromobiphenyls. The yields of target macrocycles obtained according to two routes are compared and the advantage of the approach via bis(polyamine) substituted biphenyls in the majority of cases is established. Side products, when formed in all reactions, were analyzed.
\end{abstract}

Keywords: Amination, macrocycles, Pd catalysis, polyamines, dibromobiphenyls

\section{Introduction}

Macrocycles containing biphenyl units are of constant interest due to their interesting coordination possibilities which arise from the combination of flexible and tunable polyoxa- and polyaza-cycles with a rigid non-planar aryl moiety. Many cyclic polyethers were formed starting from 2,2'-dihydroxybiphenyl; ${ }^{1-3}$ their coordination with organic cations like tert-butylammonium was studied, ${ }^{2}$ as well as the transport of $\mathrm{Li}, \mathrm{Na}, \mathrm{K}$ cations ${ }^{4,5}$ and of $\mathrm{Hg}\left(\mathrm{CF}_{3}\right)_{2}{ }^{6,7}$ through a liquid membrane. In such macrocycles one or two polyoxaethylene chains were attached to one biphenyl unit. Molecular structures of various 2,2'-biphenyl-based crown ethers and their 
coordination with anions have been studied. 8 ,9 Polyoxadiaminomacrocycles were also synthesized on the basis of 2,2'-disubstituted biphenyl and their coordination with primary alkylammonium salts, including chiral compounds, was investigated. ${ }^{10}$ Polyazamacrocycles with 3, 4 and 8 nitrogen atoms were studied for binding $\mathrm{Cu}^{2+}, \mathrm{Zn}^{2+}$ and $\left[\mathrm{PdCl}_{4}\right]^{2-}$ ions. ${ }^{11}$ Macrobicycles of cryptand type with $\mathrm{N}$ and $\mathrm{S}$ atoms have been shown to form complexes with $\mathrm{Cu}(\mathrm{I})$ and $\mathrm{Ag}(\mathrm{I}){ }^{12}$ Biphenyl-containing macrolactams have been tested as colorimetric sensors for nitrophenolate anions, ${ }^{13}$ and fluorescent and electrochemical sensors were elaborated on the basis of the macrocycles containing 2,2'-biphenyls substituted with amino goups in positions 4 and $4{ }^{\prime 14}$ Recently more sophisticated macrocycles like peptide-biphenyl hybrid ${ }^{15}$ and hemispherand macrocycle ${ }^{16}$ with bi- and quaterphenyl moietes have been reported. Cyclic triamides $^{17}$ as well as cyclic Schiff bases (trianglimines) ${ }^{18,19}$ are known, usually comprised of three 3,3'- or 4,4'-disubstituted biphenyls. Among them the most interesting are chiral nonracemic molecules with 1,2-diaminocyclohexane and 4,4'- and 3,4'-biphenyl fragments. ${ }^{20}$ Atropoisomeric biphosphine ligands were synthesized by functionalizing macrocycles constructed on the basis of 3,3'-biphenyls, and tested in the asymmetric hydrogenation of alkenes. $^{21} \pi$-Electron donor ${ }^{22}$ and acceptor $^{23}$ macrocyclic systems with 4,4'-biphenyls were reported, the first being tested as hosts for fused aromatic systems. There are some unusual macrocycles with long rigid units which combine two 4,4'-biphenyl fragments with four triple bonds linked through sulfur atoms which possess interesting electronic properties. ${ }^{24}$ The majority of known macrocycles based on biphenyls were synthesized using non-catalytic approaches which are often multistep procedures, however, in some cases one-step formation of macrocycles can be achieved, e.g. for the synthesis of aromatic tetrasulfonate 2,2'-biphenyl-based macrocycles. ${ }^{25}$ In some approaches biphenyl fragment was built using Pd-catalyzed coupling of two benzene moieties at the step of macrocyclization, as it was in the case of the compound with diazacrown, dipeptide and biphenyl fragments. ${ }^{26}$ Palladium catalysis was also employed for the synthesis of an unusual macrobicycle where anomalously bent 4,4'-biphenyl is incorporated into calix[4]amide. ${ }^{27}$ Another example of the application of the catalytic method for the synthesis of biphenyl-based macrocycles is zirconocene-mediated macrocyclization which afforded strained cyclophanes with two 4,4'-biphenyls. ${ }^{28}$ It was mentioned that biphenyls were incorporated in some biologically active macrocycles, e.g. tricyclic glucopeptides of vancomycin group. ${ }^{29}$ All these data envisage biphenyl-based macrocycles as valuable compounds with numerous applications.

Recently we have shown the possibility to synthesize earlier unknown family of polyazamacrocycles with $\mathrm{C}\left(\mathrm{sp}^{2}-\mathrm{N}\right)$ bonds by the Pd-catalyzed amination reaction of 4,4 '- and 3,3'-dibromobiphenyls with di- and polyamines. ${ }^{30,31}$ Macrocycles based on 3,3'-disubstituted biphenyl were obtained in rather high yields up to $40 \%$, whereas macrocycles containing 4,4'disubstituted biphenyl were formed only with the longest diamines and in tiny yields not exceeding $10 \%$ due to geometrical factors. In all cases the formation of cyclic dimers and oligomers was noted, and in many reactions these compounds were isolated in pure state. We decided to reveal the reaction paths according to which these symmetrical macrocycles 
containing two biphenyl and two polyamine units (cyclic dimers) may be formed, to elaborate particular methods for their synthesis because these molecules are of the utmost interest due to their versatile coordination properties, to find out scope and limitations of tested approaches and their dependence on the nature of starting compounds.

\section{Results and Discussion}

At first the synthesis of cyclodimers via intermediate $N, N^{\prime}$-bis(bromobiphenyl) substituted polyamines was studied. For the first experiments with 4,4'-dibromobiphenyl 1 we chose 1,3propanediamine $\mathbf{2 a}$, dioxadiamine $\mathbf{2} \mathbf{b}$, trioxadiamine $\mathbf{2 c}$, and tetraamine $\mathbf{2} \mathbf{d}$ as model polyamines. The reactions were run with 2.2 equivalents of compound 1 in boiling dioxane at $\mathrm{c}=0.1 \mathrm{M}$, and were catalyzed with $4 \mathrm{~mol} \% \mathrm{Pd}(\mathrm{dba})_{2} / \mathrm{BINAP}$ (Scheme 1). BINAP was taken as a ligand because<smiles>Brc1ccc(-c2ccc(Br)cc2)cc1</smiles>

2.2 equiv.
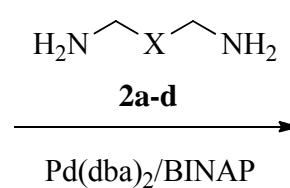

$4 / 4.5 \mathrm{~mol} \%$

$$
\text { (1) }
$$
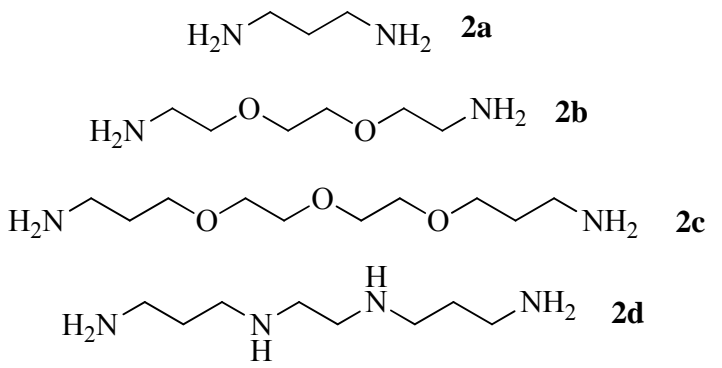

By-products:
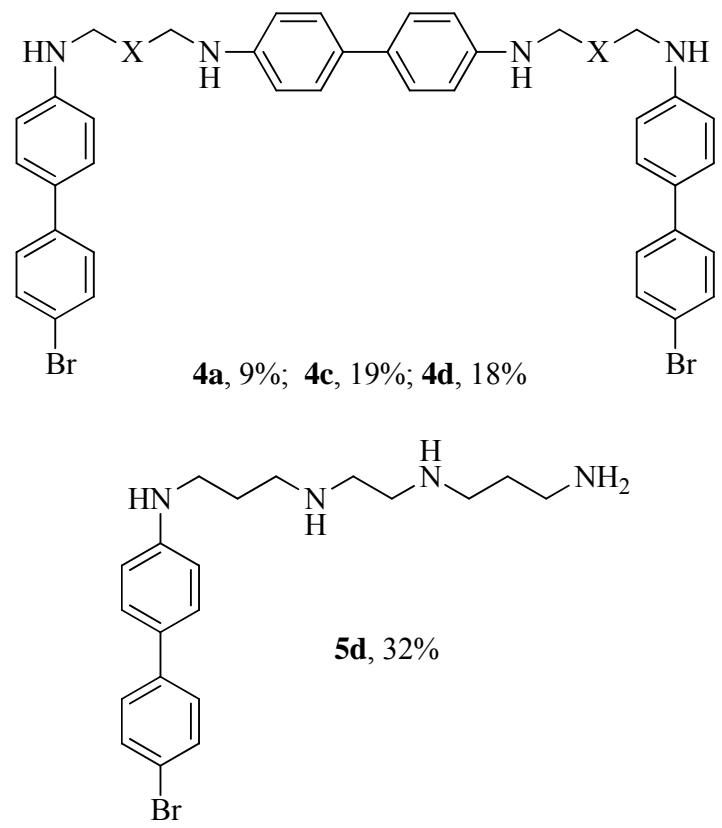

\section{Scheme 1}

it was found to be the most versatile for the Pd-mediated amination of aryl halides in general, ${ }^{32}$ and the best ligand for the diarylation of linear polyamines in particular. ${ }^{33}$ In $10 \mathrm{~h}$ the diarylation was completed and the reaction mixtures were evaporated and subjected to column chromatography on silica gel. The yields of the target compounds 3a-d after purification were not high due to the formation of linear oligomers like 4 , which were formed due to the diamination of one biphenyl fragment in compounds 3. However, it was impossible to increase the yields of the desired diarylated polyamines $\mathbf{3}$ either by decreasing the catalyst loading or by 
the application of a greater excess of $\mathbf{1}$ : in the first case the diarylation process would not come to completion, in the second case the N,N-diarylation process would take place giving rise to triarylated species. Compound $\mathbf{3 b}$ was obtained in pure state by washing the reaction mixture with small amounts of dichloromethane because its solubility was found to be unusually low. In one case, with tetraamine 3d, the diarylation process was not completed, and monoarylated derivative $5 \mathbf{d}$ was obtained in $32 \%$ yield.

The formation of the macrocycles 6a-d was accomplished by the reactions of $N, N^{\prime}-$ bis(bromobiphenyl) polyamines 3a-d with one equivalent of corresponding di- and polyamines 2a-d, which were catalyzed by $8 \mathrm{~mol} \% \mathrm{Pd}(\mathrm{dba})_{2} / \mathrm{BINAP}$ (Scheme 2 ). In these processes more dilute solutions were employed $(\mathrm{c}=0.02 \mathrm{M})$ to suppress the formation of linear and cyclic oligomers. The reactions ran to completion after $30-40 \mathrm{~h}$ of reflux. Macrocycles with trioxadiamine and tetraamine chains $\mathbf{6 c , d}$ were isolated in 20 and 23\% yields, respectively, while the yield of the macrocycle with dioxadiamine unit was small $(9 \%)$, and the shortest $1,3-$ propanediamine 2a gave a mixture of the cyclic dimer $\mathbf{6 a}$ with cyclotetramer $\mathbf{7 a}(\mathrm{n}=4)$ in total $6 \%$ yield. In the reaction of compound $4 \mathrm{c}$ with trioxadiamine 2c the formation of cyclic oligomers was also noted, and the mixture of $\mathbf{6 c}$ with $7 \mathbf{c}(\mathrm{n}=4,6)$ was isolated by column chromatography as a separate fraction. In this reaction the product of the bromine reduction $\mathbf{8 c}$ was obtained in $16 \%$ yield, though the reduction processes are not common in the Pd-catalyzed amination with di- and polyamines. We also tried to accomplish the synthesis of cyclodimers via a one-step procedure without isolation of the intermediate diaryl derivatives 3 .<smiles>[X]CNc1ccc(-c2ccc(Br)cc2)cc1</smiles>

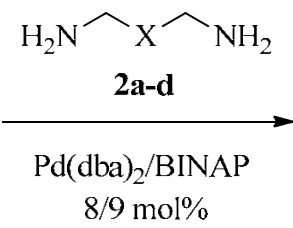

3a-d

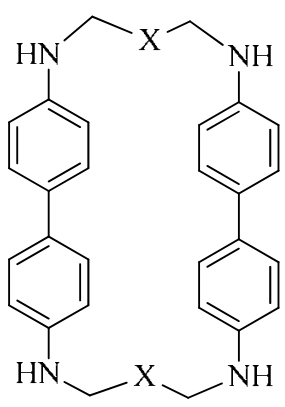

6b, $9 \%$

6c, $20 \%$

6d, $23 \%$

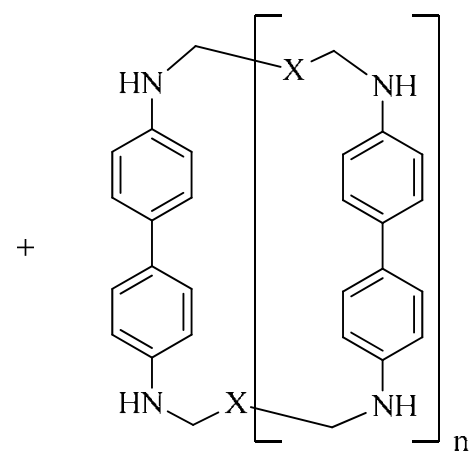

$6 \mathbf{a}+7 \mathbf{a}(\mathrm{n}=3), 6 \%$

$\mathbf{6 c}+7 \mathbf{c}(\mathrm{n}=3,5), 23 \%$

From in situ obtained 3c: $\quad$ 6c, $5 \%$

$\mathbf{6 c}+7 \mathbf{c}(\mathrm{n}=2), 9 \%$

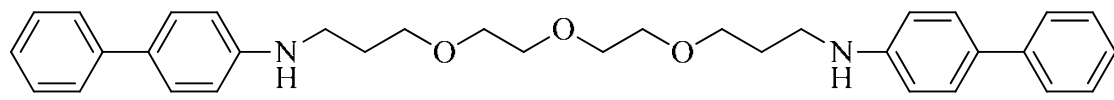

8c, $16 \%$ (from isolated $3 \mathrm{c}$ ), $28 \%$ (from in situ obtained $3 \mathrm{c}$ )

\section{Scheme 2}


For this purpose 2.5 equivalents of 4,4'-dibromobiphenyl were reacted with trioxadiamine 2c using $4 \mathrm{~mol} \%$ catalyst at $\mathrm{c}=0.1 \mathrm{M}$. After $10 \mathrm{~h}$ the reaction mixture was diluted to get $0.02 \mathrm{M}$ solution, additional amount of the catalyst $(8 \mathrm{~mol} \%)$ and one equivalent of trioxadiamine 2c were added, and the reaction was refluxed for $35 \mathrm{~h}$. The chromatography gave $28 \%$ of the reduction product $8 \mathrm{c}$, only $5 \%$ of pure cyclic dimer $\mathbf{6 c}$, and a separate fraction containing cyclodimer $\mathbf{6 c}$ with cyclic trimer $7 c(n=3)(9 \%$ yield). This unsatisfactory result demonstrates the difficulties in the separation of cyclic oligomers and thus the pressure to diminish their amount and diversity, which can be done only using pure $N, N^{\prime}$-bis(4-biphenyl) derivatives of polyamines for the synthesis of cyclic dimers.

While macrocycles based on 4,4'-biphenyls are well documented in the literature, macrocycles incorporating 3,3'-biphenyls are quite rare examples, and this fact makes the synthesis of cyclic dimers with two 3,3'-biphenyl moieties an important task. At first we tried a one-step approach described above without isolation of the intermediate N,N'-diarylated derivatives of polyamines (Scheme 3).
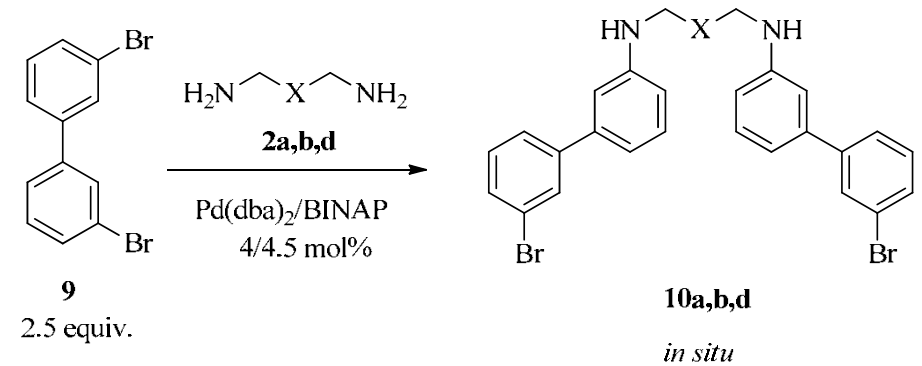

10a,b,d

in situ

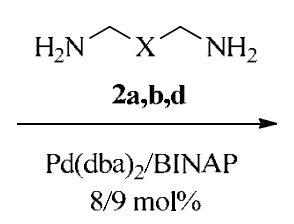

$8 / 9 \mathrm{~mol} \%$

$$
\text { By-products: }
$$

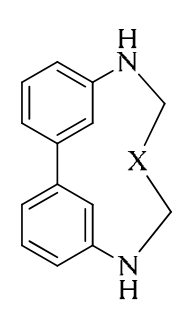

$12 \mathrm{~b}, 10 \%$ 12d, $18 \%$

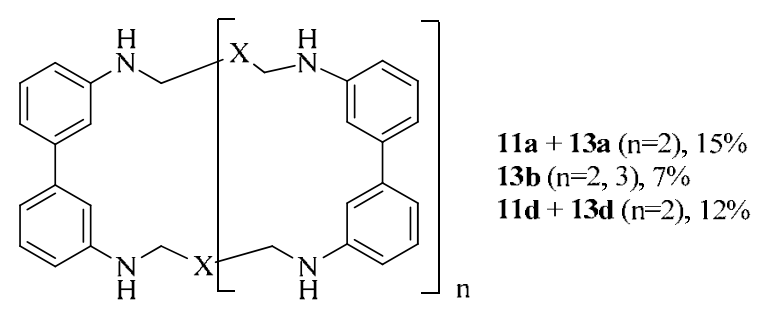

$13, n=2,3$<smiles>[X]CNCNc1cccc(-c2cccc(NC[X])c2)c1</smiles>

\section{Scheme 3}

N,N'-diarylation of polyamines 2a,b,d was achieved using 2.5 equivalents of 3,3'dibromobiphenyl 9, $4 \mathrm{~mol} \%$ catalyst, and $0.1 \mathrm{M}$ solutions of polyamines in dioxane. After $10 \mathrm{~h}$ of reflux the reaction mixtures were analyzed by ${ }^{1} \mathrm{H}$ NMR to verify the formation of diaryl derivatives 10, they were diluted to make $0.02 \mathrm{M}$ solutions and new portions of the catalyst (8 mol\%) were added. The additional reflux for $35-40 \mathrm{~h}$ was necessary to complete the intramolecular diamination, and after it the reaction mixtures were subjected to chromatography on silica gel. The result was moderate only for tetraamine derivative 11d (19\%), while in other 
cases pure cyclodimers 11a,b were obtained in low yields. Cyclic oligomers 13 were isolated in all reactions, and in the case of dioxadiamine $\mathbf{2} \mathbf{b}$ and tetraamine $\mathbf{2 d}$ also macrocycles $\mathbf{1 2} \mathbf{b}, \mathbf{d}$ were obtained in comparable yields (10 and $18 \%$, respectively). The reaction of equimolar amounts of 1,3-diaminopropane 2a with 3,3'-dibromobiphenyl 9 described by us previously afforded 24\% yield of the cyclic dimer 11a. ${ }^{31}$ Thus it is clear that the approach via in situ obtained $N, N^{\prime}$-bis(3bromobiphenyl)diamine is not suitable for this compound.

Next we investigated the possibilities of this method with chromatographic isolation of compounds 10; this was thought also to reveal the formation of by-products (Scheme 4).<smiles>Brc1cccc(-c2cccc(Br)c2)c1</smiles>

9

2.2-3 equiv.

By-products:<smiles>[X]CNc1cccc(-c2cccc(NC[X])c2)c1</smiles>

12c, $11 \%(\mathrm{~L}=$ BINAP, 2,2 equiv. of 9) 12d, 7\% ( $\mathrm{L}=$ BINAP, 2,2 equiv. of 9$)$<smiles>[X]CNc1cccc(NCCNc2cccc(-c3cccc(Br)c3)c2)c1</smiles>

$$
\begin{gathered}
\mathbf{1 0 b}, 27 \%(\mathrm{~L}=\text { Xanthphos, } 3 \text { equiv. of } \mathbf{9}) \\
\mathbf{1 0 c}, 21 \%(\mathrm{~L}=\text { BINAP, } 2,2 \text { equiv. of } \mathbf{9}) \\
35 \%(\mathrm{~L}=\text { Xanthphos, } 3 \text { equiv. of } \mathbf{9}) \\
\mathbf{1 0 d}, 26 \%(\mathrm{~L}=\text { BINAP, } 2.2 \text { equiv. of } \mathbf{9}) \\
34 \%(\mathrm{~L}=\text { BINAP, } 3 \text { equiv. of } 9)
\end{gathered}
$$<smiles>[X]CCNc1cccc(-c2cccc(NCCC)c2)c1</smiles>

14b-d $(n=1), 15 d(n=2)$
$14 \mathrm{~b}, 5 \%(\mathrm{~L}=$ Xanthphos, 3 equiv. of 9$)$

$14 c, 11 \%(\mathrm{~L}=$ BINAP, 2,2 equiv. of 9) $19 \%(\mathrm{~L}=$ Xanthphos, 3 equiv. of 9$)$

14d, 28\% ( $\mathrm{L}=$ BINAP, 2,2 equiv. of 9)

$32 \%(\mathrm{~L}=$ BINAP, 3 equiv. of 9$)$

$15 d, 12 \%(L=B I N A P, 2,2$ equiv. of 9$)$

\section{Scheme 4}

According to a given consideration, diamine 2a was excluded from this series of experiments. The reactions were run using 2.2 equivalents of $\mathbf{9}$, and at first they were catalyzed by $4 \mathrm{~mol} \% \mathrm{Pd}(\mathrm{dba})_{2} / \mathrm{BINAP}$. The reactions of trioxadiamine 2c and tetraamine $2 \mathrm{~d}$ gave target 
products 10c,d in 21 and 26\% yields, macrocycles 12c,d as well as linear oligomers 14c,d and 15d were also obtained. In the case of tetraamine 2d the application of 3 equivalents of $\mathbf{9}$ allowed to increase the yield of $\mathbf{1 0 d}$ to $34 \%$. However, for dioxadiamine $\mathbf{2 b}$ it was found impossible to isolate the target compound 10b in pure state because it contained admixtures - products of N,Ndiarylation and diamination reactions. The application of less amount of the catalyst ( $2 \mathrm{~mol} \%)$ did not improve the situation. The change of BINAP ligand for a less active Xanthphos suppressed the undesirable reactions but the standard catalyst loading ( 4 mol\%) together with the standard reaction time $(10 \mathrm{~h})$ led mainly to monoaryl derivative of dioxadiamine. The use of more catalyst ( $8 \mathrm{~mol} \%$ ) was unsuccessful because it promoted $\mathrm{N}, \mathrm{N}$-diarylation process, but the employment of 3 equivalents of 9 and prolonged reflux ( $45 \mathrm{~h})$ with $4 \mathrm{~mol} \% \mathrm{Pd}(\mathrm{dba})_{2} / \mathrm{Xanthphos}$ resulted in $27 \%$ yield of pure product $\mathbf{1 0 b}$. We tested the same conditions with trioxadiamine 2c and increased the yield of 10c to $35 \%$.

The cyclization reactions of diaryl derivatives 10b-d were accomplished under standard conditions (Scheme 5) and their results were similar: the yields of target macrocycles 12b-d ranged from 25 to $30 \%$, cyclic oligomers (cyclotetramers and cyclohexamers) were obtained as side products in comparable yields. In the case of the reaction of $\mathbf{1 0 b}$ with $\mathbf{2} \mathbf{b}$ cyclooligomers 13b $(n=3,5)$ were fully separated from the target cyclodimer 11b, while in other reactions the fractions containing cyclooligomers 13c,d contained also cyclodimers 11c,d what to some extent diminished the yields of the target macrocycles-cyclodimers. This macrocyclization was more successful than that with $N, N^{\prime}$-bis(4-bromobiphenyl) derivatives $\mathbf{3}$ and here no influence of the nature of polyamine was noted.

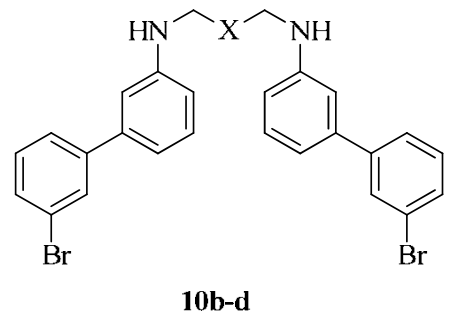

10b-d

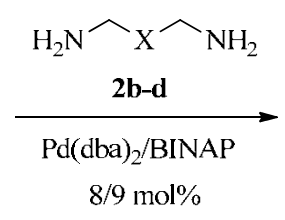

$8 / 9 \mathrm{~mol} \%$

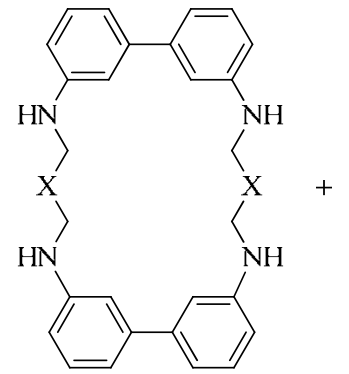

11b, $27 \%$

11c, $30 \%$

11d, $25 \%$

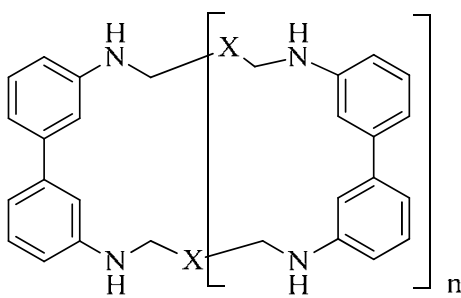

$13 \mathbf{b}(\mathrm{n}=3), 13 \% ; \mathbf{1 3 b}(\mathrm{n}=3,5), 18 \%$ $11 \mathrm{c}+13 \mathbf{c}(\mathrm{n}=3,5), 48 \%$ $11 d+13 d(n=3), 28 \%$

\section{Scheme 5}

Having obtained data on the synthesis of the biphenyl-containing macrocycles via intermediate $N, N^{\prime}$-bis(bromobiphenyl)polyamines, we tried an alternative approach, i.e. via intermediate bis(polyamine) substituted biphenyls. In this method intermediates were not isolated by column chromatography due to great difficulties caused by the presence of an excess 
of polyamines in the reaction mixture which possess very close $R_{f}$ to that of bis(polyamine) derivatives of biphenyls.

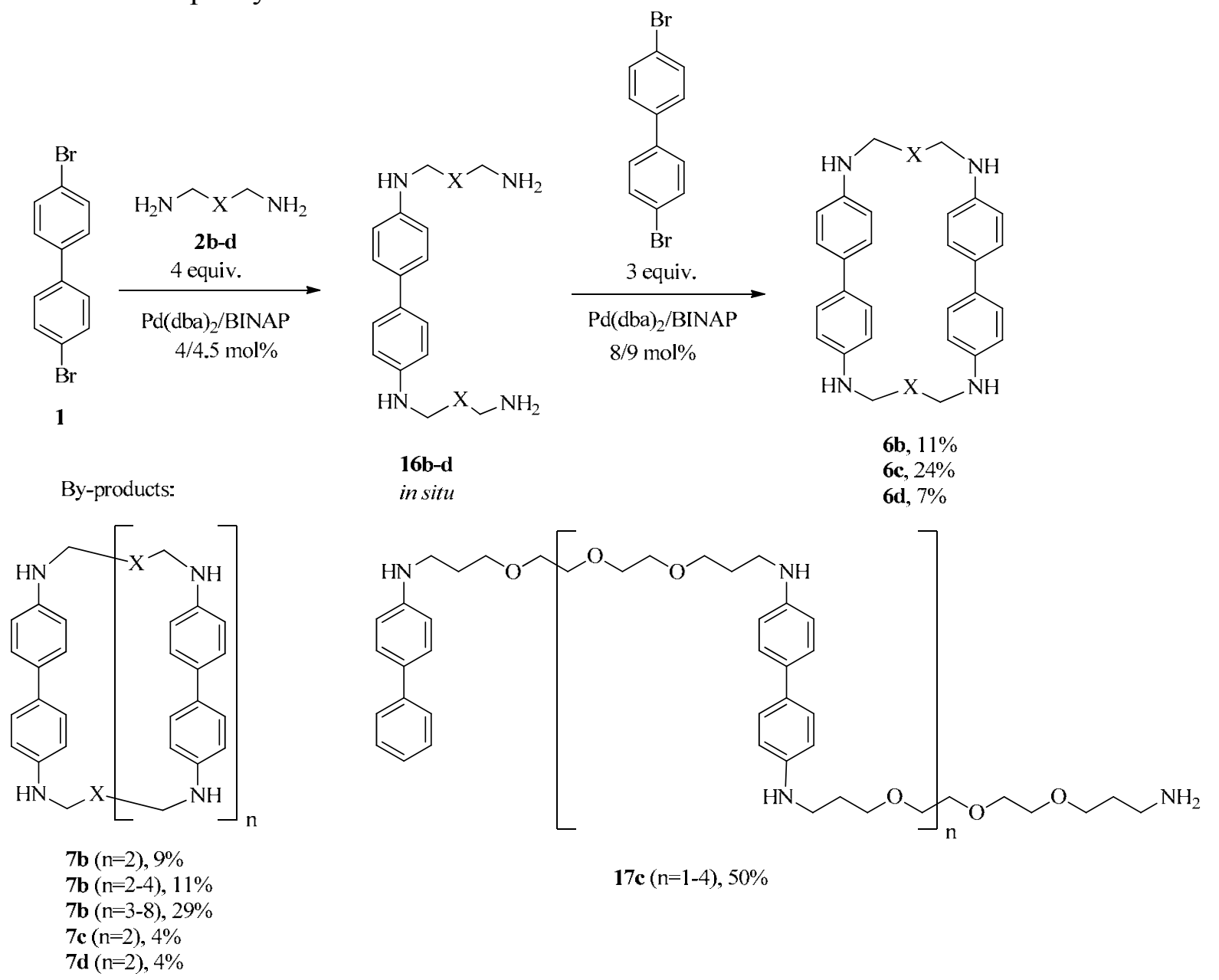

Scheme 6

The reactions of 4,4'-dibromobiphenyl 1 with 4 equivalents of di- and polyamines $\mathbf{2 b}$-d were run in boiling dioxane $(\mathrm{C}=0.1 \mathrm{M})$ and catalyzed by $8 \mathrm{~mol} \% \mathrm{Pd}(\mathrm{dba})_{2} / \mathrm{BINAP}$, they were complete in 8-10 h (Scheme 6). Intermediate 4,4'-bis(polyamine) biphenyls 16a-d were analyzed by NMR and MALDI-TOF mass-spectra in the reaction mixtures. Then 3 equivalents of 4,4'dibromobiphenyl, additional catalyst $(8 \mathrm{~mol} \%)$ and dioxane to make $0.02 \mathrm{M}$ solution were added, and the reactions were refluxed for 35-40 h. Target cyclodimers $\mathbf{6 b - d}$ were isolated by column chromatography on silica gel. The best result was obtained for the macrocycle 6c $(24 \%)$, the reactions with dioxadiamine $\mathbf{2} \mathbf{b}$ and tetraamine $\mathbf{2 d}$ gave poor yields of macrocycles (11 and 7\% respectively). In these reactions substantial amounts of cyclic oligomers of higher masses were obtained (up to cyclononamer $\mathbf{7 b}(\mathrm{n}=8)$ ), obviously, they were formed via intermediate linear oligomers containing $n$ biphenyl and $n+1$ polyamine units. It is notable that the reaction with trioxadiamine 2c gave rise to linear oligomers 17c with terminal biphenyl which were formed due to the reduction of the bromine atom. 
A similar reaction with the isomeric 3,3'-dibromobiphenyl 9 conducted under the same conditions gave somewhat different results (Scheme 7).

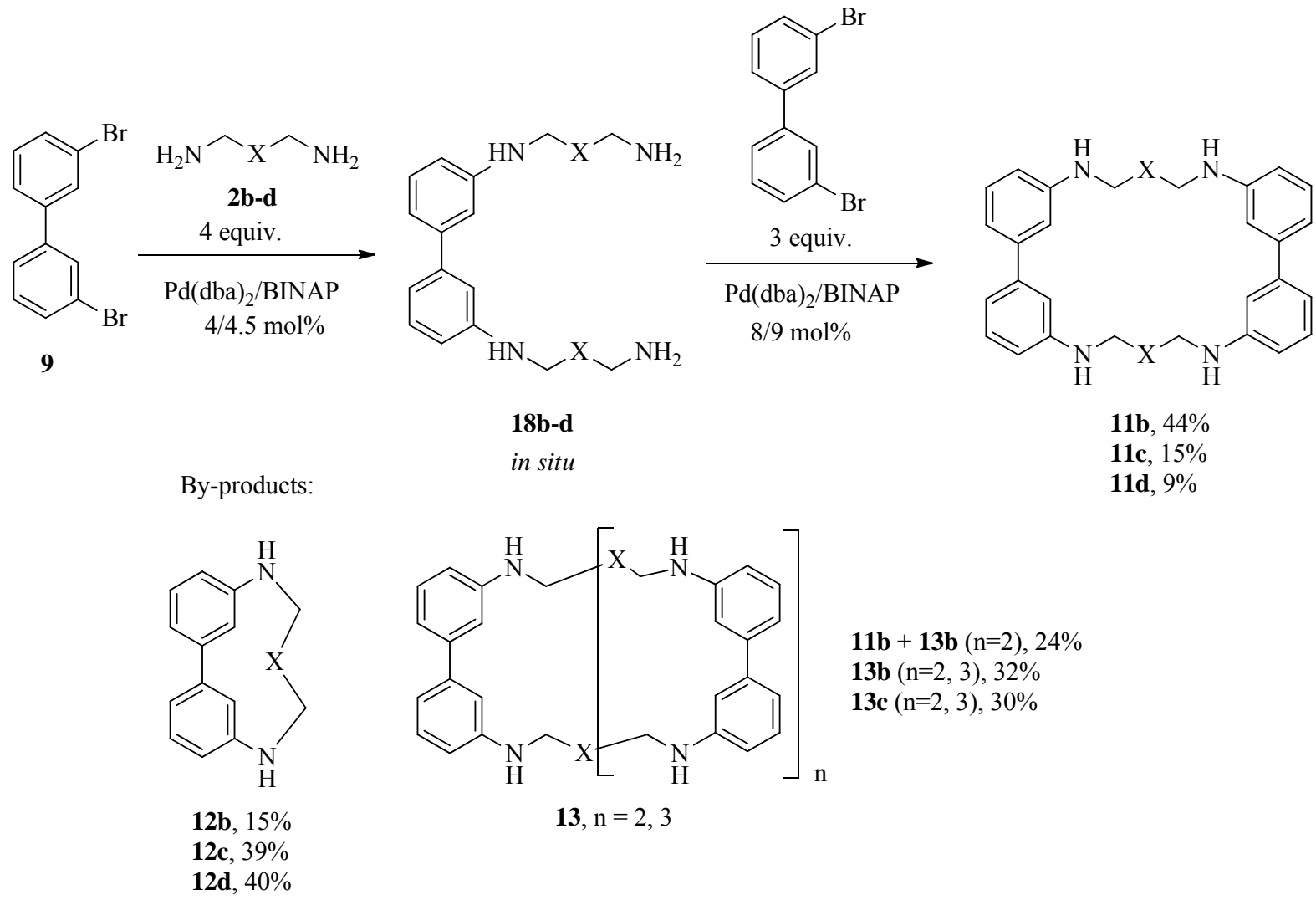

\section{Scheme 7}

Intermediate 3,3'-bis(polyamine)biphenyls $\mathbf{1 8 b}$-d, obtained in situ using 4 equivalents of corresponding di- and polyamines $\mathbf{2 b - d}$, were analyzed by NMR and MALDI-TOF spectroscopy, and they were further reacted with 3 equivalents of 3,3'-dibromobiphenyl. The best result was recorded for the macrocycle 11b with its highest $44 \%$ yield, whereas the yields of compounds 11c and 11d were much more modest (15 and 9\% respectively). In all cases the formation of the macrocycles 12 containing one set of biphenyl and polyamine units was observed, and with longer trioxadiamine 2c and tetraamine 2d their yields were about $40 \%$ which is equal to the yields of these compounds when the reactions were run with equimolar amounts of starting compounds. ${ }^{31}$ Higher mass cyclooligomers were also formed in the case of dioxadiamine $\mathbf{2 b}$ and trioxadiamine $\mathbf{2 c}$.

\section{Conclusions}

As a result of the experiments described above, we can outline the following general findings. The synthesis of the macrocycles containing two biphenyl and two polyamine moieties can be 
carried out using two approaches: via $N, N^{\prime}$-bis(bromobiphenyl) substituted polyamines or via bis(polyamine)biphenyls. The first route in a two-step version affords target macrocycles in yields up to $30 \%, 3,3$ '-dibromobiphenyl providing better yields of the macrocycles than 4,4'dibromobiphenyl. One-step version with in situ obtained $N, N^{\prime}$-bis(bromobiphenyl) derivative can be recommended only for the synthesis of the cyclodimer with two 3,3'-biphenyls and two tetraamine chains (yield 19\%). The second approach via bis(polyamine)biphenyls provides better yields of some cyclodimers, and in this case the dependence of the result on the nature of starting compounds is pronounced: 4,4'-dibromobiphenyl gave $24 \%$ yield of the macrocycle with two trioxadiamine chains, whereas 3,3'-dibromobiphenyl afforded the cyclodimer with dioxadiamine in the highest yield (44\%). We suppose that two main factors govern the results of these two-step syntheses: the conformational factors of di- and polyamine chains at the macrocyclization step and the activity of the bromine atom in 4,4'- and 3,3'-dibromobiphenyls in competitive amination and reduction reactions at both steps of the processes. The application of more powerful separation techniques like HPLC may undoubtedly increase the yields of the target macrocycles.

\section{Experimental Section}

General. All chemicals were purchased from Aldrich and Acros companies and used without further purification. 3,3'-Dibromobiphenyl 9 was synthesized from 2-bromonitrobenzene according to a procedure described in ref. 34. $\mathrm{Pd}(\mathrm{dba})_{2}$ was synthesized according to a described method. $^{35}$ Commercial dioxane was distilled over $\mathrm{NaOH}$ and sodium under argon, dichloromethane and methanol were distilled prior to use. Column chromatography was carried out using silica gel (40-60 mkm) purchased from Fluka. ${ }^{1} \mathrm{H}$ and ${ }^{13} \mathrm{C}$ NMR spectra were registered in $\mathrm{CDCl}_{3}$ using Bruker Avance 400 spectrometer at 400 and $100.6 \mathrm{MHz}$ respectively. Chemical shift values $\delta$ are given in ppm and coupling constants $J$ in Hz. MALDI-TOF mass spectra of positive ions were recorded with Briker Ultraflex spectrometer using 1,8,9-trihydroxyanthracene as matrix and PEGs as internal standards. Spectral data for compounds $\mathbf{6 b}, \mathbf{c}, \mathbf{7 c}(\mathrm{n}=2)$ are given in ref. 30, and spectral data for compounds 11a-d, 12b-d and 13c $(n=2)$ are given in ref. 31 .

Standard method for the synthesis of $N, N^{\prime}$-bis(bromobiphenyl)polyamines 3a-d and 10b-d. A two-neck flask equipped with a magnetic stirrer, flushed with dry argon was charged with 4,4'dibromobiphenyl 1 or 3,3'-dibromobiphenyl 9 (2.2 mmol, $686 \mathrm{mg}), \operatorname{Pd}(\mathrm{dba})_{2}(0.04 \mathrm{mmol}, 23$ $\mathrm{mg})$, BINAP $(0.045 \mathrm{mmol}, 28 \mathrm{mg})$, absolute dioxane $(10 \mathrm{ml})$, the mixture was stirred for $2 \mathrm{~min}$, then appropriate polyamine $\mathbf{2 a - d}(1 \mathrm{mmol})$ was added, followed by $t \mathrm{BuONa}(3 \mathrm{mmol}, 290 \mathrm{mg})$. The reaction mixture was refluxed for $10 \mathrm{~h}$, cooled to ambient temperature, filtered and evaporated in vacuo. The residue was chromatographed on silica gel using a sequence of eluents: $\mathrm{CH}_{2} \mathrm{Cl}_{2}, \mathrm{CH}_{2} \mathrm{Cl}_{2} / \mathrm{MeOH}$ 500:1 - 3:1, $\mathrm{CH}_{2} \mathrm{Cl}_{2} / \mathrm{MeOH} / \mathrm{NH}_{3}$ aq 100:20:1 - 10:4:1.

$\boldsymbol{N}^{\mathbf{1}}, \boldsymbol{N}^{\mathbf{3}}$-Bis(4'-bromobiphenyl-4-yl)propane-1,3-diamine (3a) was synthesized from 1,3propanediamine (2a) (74 mg). Eluent $\mathrm{CH}_{2} \mathrm{Cl}_{2}, \mathrm{CH}_{2} \mathrm{Cl}_{2}-\mathrm{MeOH}$ 500:1, yield $135 \mathrm{mg}$ (25\%), pale- 
yellow crystals, m.p. $209-210^{\circ} \mathrm{C} .{ }^{1} \mathrm{H}$ NMR $\delta 1.99$ (quintet, ${ }^{3} \mathrm{~J}=6.7 \mathrm{~Hz}, 2 \mathrm{H}$ ), $3.32\left(\mathrm{t},{ }^{3} \mathrm{~J}=6.7 \mathrm{~Hz}\right.$, $4 \mathrm{H}), 6.68\left(\mathrm{~d},{ }^{3} \mathrm{~J}=8.7 \mathrm{~Hz}, 4 \mathrm{H}\right), 7.38\left(\mathrm{~d},{ }^{3} J=8.4 \mathrm{~Hz}, 4 \mathrm{H}\right), 7.40\left(\mathrm{~d},{ }^{3} J=8.7 \mathrm{~Hz}, 4 \mathrm{H}\right), 7.49\left(\mathrm{~d},{ }^{3} J=\right.$ $8.4 \mathrm{~Hz}, 4 \mathrm{H}), \mathrm{NH}$ protons were not assigned; ${ }^{13} \mathrm{C}$ NMR $\delta 29.1$ (1C), $42.0(2 \mathrm{C}), 113.3$ (4C), 120.0 (2C), 127.8 (8C), 129.0 (2C), 131.7 (4C), 140.0 (2C), 147.6 (2C); HRMS MALDI-TOF m/z: calcd. for $\mathrm{C}_{27} \mathrm{H}_{25} \mathrm{Br}_{2} \mathrm{~N}_{2} 535.0384[\mathrm{M}+\mathrm{H}]^{+}$, found 535.0359.

$N^{1}, N^{1}$-(Biphenyl-4,4'-diyl)bis( $N^{3}$-(4'-bromobiphenyl-4-yl)propane-1,3-diamine) (4a) was obtained as the second product in the synthesis of compound 3a. Eluent $\mathrm{CH}_{2} \mathrm{Cl}_{2}-\mathrm{MeOH} 200: 1$, yield $35 \mathrm{mg}\left(9 \%\right.$ ), pale-yellow glassy solid. ${ }^{1} \mathrm{H}$ NMR $\delta 1.98$ (quintet, ${ }^{3} \mathrm{~J}=6.6 \mathrm{~Hz}, 4 \mathrm{H}$ ), $3.31\left(\mathrm{t},{ }^{3} \mathrm{~J}\right.$ $=6.4 \mathrm{~Hz}, 4 \mathrm{H}), 3.32\left(\mathrm{t},{ }^{3} \mathrm{~J}=6.4 \mathrm{~Hz}, 4 \mathrm{H}\right), 3.80(\mathrm{br} \mathrm{s}, 2 \mathrm{H}), 3.91(\mathrm{br} \mathrm{s}, 2 \mathrm{H}), 6.66\left(\mathrm{~d},{ }^{3} \mathrm{~J}=8.5 \mathrm{~Hz}\right.$, $4 \mathrm{H}), 6.67\left(\mathrm{~d},{ }^{3} \mathrm{~J}=8.5 \mathrm{~Hz}, 4 \mathrm{H}\right), 7.35-7.42(\mathrm{~m}, 12 \mathrm{H}), 7.49\left(\mathrm{~d},{ }^{3} \mathrm{~J}=8.2 \mathrm{~Hz}, 4 \mathrm{H}\right) ;{ }^{13} \mathrm{C}$ NMR $\delta 29.2$ (2C), 41.9 (2C), 42.1 (2C), 113.1 (4C), 113.2 (4C), 120.0 (2C), 127.2 (4C), 127.8 (8C), 129.0 (2C), 131.7 (4C), 132.0 (2C), 140.1 (2C), 146.7 (2C), 147.8 (2C); HRMS MALDI-TOF m/z: calcd. for $\mathrm{C}_{42} \mathrm{H}_{41} \mathrm{Br}_{2} \mathrm{~N}_{4} 759.1698[\mathrm{M}+\mathrm{H}]^{+}$, found 759.1662 .

$N, N^{\prime}$-(2,2'-(Ethane-1,2-diylbis(oxy))bis(ethane-2,1-diyl))bis(4'-bromobiphenyl-4-amine)

(3b) was synthesized from dioxadiamine $2 \mathbf{b}(148 \mathrm{mg})$. Isolated from the reaction mixture by treatment with $\mathrm{CH}_{2} \mathrm{Cl}_{2}$. Pale-yellow crystals, m.p. $184-185^{\circ} \mathrm{C} .{ }^{1} \mathrm{H}$ NMR $\delta 3.34\left(\mathrm{t},{ }^{3} \mathrm{~J}=5.2 \mathrm{~Hz}\right.$, 4H), $3.67(\mathrm{~s}, 4 \mathrm{H}), 3.73\left(\mathrm{t},{ }^{3} \mathrm{~J}=5.2 \mathrm{~Hz}, 4 \mathrm{H}\right), 4.21(\mathrm{br} \mathrm{s}, 2 \mathrm{H}), 6.65\left(\mathrm{~d},{ }^{3} \mathrm{~J}=8.6 \mathrm{~Hz}, 4 \mathrm{H}\right), 7.35\left(\mathrm{~d},{ }^{3} \mathrm{~J}\right.$ $=8.7 \mathrm{~Hz}, 8 \mathrm{H}), 7.47\left(\mathrm{~d},{ }^{3} J=8.3 \mathrm{~Hz}, 4 \mathrm{H}\right) ;{ }^{13} \mathrm{C}$ NMR $\delta 43.4(2 \mathrm{C}), 69.6(2 \mathrm{C}), 70.3(2 \mathrm{C}), 113.3(4 \mathrm{C})$, 120.0 (2C), 127.7 (4C), 127.8 (4C), 129.0 (2C), 131.7 (4C), 140.1 (2C), 147.9 (2C); HRMS MALDI-TOF $\mathrm{m} / \mathrm{z}$ : calcd. for $\mathrm{C}_{30} \mathrm{H}_{31} \mathrm{Br}_{2} \mathrm{~N}_{2} \mathrm{O}_{2} 609.0752[\mathrm{M}+\mathrm{H}]^{+}$, found 609.0784 .

$N, N^{\prime}-(3,3$ '-(2,2'-Oxybis(ethane-2,1-diyl)bis(oxy))bis(propane-3,1-diyl))bis(4'-bromobi

phenyl-4-amine) (3c) was synthesized from trioxadiamine 2c (220 mg). Eluent $\mathrm{CH}_{2} \mathrm{Cl}_{2}-\mathrm{MeOH}$ 200:1-100:1, yield $235 \mathrm{mg}$ (34\%), pale-yellow crystals, m.p. 104-105 ${ }^{\circ} \mathrm{C} .{ }^{1} \mathrm{H}$ NMR $\delta 1.91$ (quintet, $\left.{ }^{3} J=6.1 \mathrm{~Hz}, 4 \mathrm{H}\right), 3.27\left(\mathrm{t},{ }^{3} J=6.5 \mathrm{~Hz}, 4 \mathrm{H}\right), 3.63\left(\mathrm{t},{ }^{3} J=5.8 \mathrm{~Hz}, 4 \mathrm{H}\right), 3.62-3.66(\mathrm{~m}, 4 \mathrm{H})$, 3.69-3.73 (m, 4H), $4.23(\mathrm{br} \mathrm{s}, 2 \mathrm{H}), 6.65\left(\mathrm{~d},{ }^{3} \mathrm{~J}=8.5 \mathrm{~Hz}, 4 \mathrm{H}\right), 7.38\left(\mathrm{~d},{ }^{3} \mathrm{~J}=8.7 \mathrm{~Hz}, 4 \mathrm{H}\right), 7.39(\mathrm{~d}$, $\left.{ }^{3} \mathrm{~J}=8.5 \mathrm{~Hz}, 4 \mathrm{H}\right), 7.49\left(\mathrm{~d},{ }^{3} \mathrm{~J}=8.7 \mathrm{~Hz}, 4 \mathrm{H}\right) ;{ }^{13} \mathrm{C}$ NMR $\delta 28.9(2 \mathrm{C}), 41.7(2 \mathrm{C}), 69.7(2 \mathrm{C}), 70.2$ (2C), 70.5 (2C), 112.9 (4C), 119.7 (2C), 127.5 (4C), 127.6 (4C), 128.3 (2C), 131.6 (4C), 140.1 (2C), 148.1 (2C); HRMS MALDI-TOF m/z: calcd. for $\mathrm{C}_{34} \mathrm{H}_{39} \mathrm{Br}_{2} \mathrm{~N}_{2} \mathrm{O}_{3} 681.1327[\mathrm{M}+\mathrm{H}]^{+}$, found 681.1345 .

$N^{4}, N^{4}$-Bis[3-[2-[2-[3-(4'-bromobiphenyl-4-ylamino)propoxy]ethoxy]ethoxy]propyl]-bi phenyl-4,4'-diamine (4c) was obtained as the second product in the synthesis of compound 3c. Eluent $\mathrm{CH}_{2} \mathrm{Cl}_{2}-\mathrm{MeOH}$ 50:1, yield $102 \mathrm{mg}$ (19\%), pale-yellow glassy solid. ${ }^{1} \mathrm{H}$ NMR $\delta 1.90$ (quintet, $\left.{ }^{3} J=6.1 \mathrm{~Hz}, 8 \mathrm{H}\right), 3.25\left(\mathrm{t},{ }^{3} J=6.3 \mathrm{~Hz}, 4 \mathrm{H}\right), 3.26\left(\mathrm{t},{ }^{3} \mathrm{~J}=6.3 \mathrm{~Hz}, 4 \mathrm{H}\right), 3.61\left(\mathrm{t},{ }^{3} J=5.9 \mathrm{~Hz}\right.$, $8 \mathrm{H}), 3.61-3.65(\mathrm{~m}, 8 \mathrm{H}), 3.67-3.71(\mathrm{~m}, 8 \mathrm{H}), 4.07(\mathrm{br} \mathrm{s}, 4 \mathrm{H}), 6.64\left(\mathrm{~d},{ }^{3} \mathrm{~J}=8.4 \mathrm{~Hz}, 4 \mathrm{H}\right), 6.65\left(\mathrm{~d},{ }^{3} J\right.$ $=8.6 \mathrm{~Hz}, 4 \mathrm{H}), 7.33-7.41(\mathrm{~m}, 12 \mathrm{H}), 7.48\left(\mathrm{~d},{ }^{3} \mathrm{~J}=8.5 \mathrm{~Hz}, 4 \mathrm{H}\right) ;{ }^{13} \mathrm{C}$ NMR $\delta 28.9(2 \mathrm{C}), 29.0(2 \mathrm{C})$, 41.5 (2C), 41.8 (2C), 69.6 (4C), 70.1 (4C), 70.5 (4C), 112.8 (4C), 113.0 (4C), $119.6(2 \mathrm{C}), 126.8$ (4C), 127.4 (4C), 127.5 (4C), 128.5 (2C), 130.2 (2C), 131.5 (4C), 140.1 (2C), 146.8 (2C), 148.2 (2C); HRMS MALDI-TOF m/z: calcd. for $\mathrm{C}_{56} \mathrm{H}_{69} \mathrm{Br}_{2} \mathrm{~N}_{4} \mathrm{O}_{6} 1051.3584[\mathrm{M}+\mathrm{H}]^{+}$, found 1051.3532 . $N^{1}, N^{1^{\prime}}$-(Ethane-1,2-diyl)bis[ $N^{3}$-(4'-bromobiphenyl-4-yl)propane-1,3-diamine] (3d) was synthesized from tetraamine 2d (174 mg). Eluent $\mathrm{CH}_{2} \mathrm{Cl}_{2}-\mathrm{MeOH}-\mathrm{NH}_{3}$ aq 100:20:1, yield $142 \mathrm{mg}$ 
(22\%), pale-yellow crystals, m.p. $158-160^{\circ} \mathrm{C} .{ }^{1} \mathrm{H}$ NMR $\delta 1.81$ (quintet, ${ }^{3} J=6.6 \mathrm{~Hz}, 4 \mathrm{H}$ ), 2.75 (s, $4 \mathrm{H}), 2.77\left(\mathrm{t},{ }^{3} \mathrm{~J}=6.6 \mathrm{~Hz}, 4 \mathrm{H}\right), 3.22\left(\mathrm{t},{ }^{3} J=6.6 \mathrm{~Hz}, 4 \mathrm{H}\right), 6.63\left(\mathrm{~d},{ }^{3} J=8.7 \mathrm{~Hz}, 4 \mathrm{H}\right), 7.36\left(\mathrm{~d},{ }^{3} J=\right.$ $8.4 \mathrm{~Hz}, 8 \mathrm{H}), 7.47\left(\mathrm{~d},{ }^{3} \mathrm{~J}=8.6 \mathrm{~Hz}, 4 \mathrm{H}\right), \mathrm{NH}$ protons were not assigned; ${ }^{13} \mathrm{C}$ NMR $\delta 29.4(2 \mathrm{C})$, 42.7 (2C), 48.2 (2C), 49.4 (2C), 112.9 (4C), 119.9 (2C), 127.7 (8C), 128.5 (2C), 131.6 (4C), 140.1 (2C), 148.2 (2C); HRMS MALDI-TOF m/z: calcd. for $\mathrm{C}_{32} \mathrm{H}_{37} \mathrm{Br}_{2} \mathrm{~N}_{4} 635.1385[\mathrm{M}+\mathrm{H}]^{+}$, found 635.1357 .

$N^{1}, N^{1}$-(Biphenyl-4,4'-diyl)bis[ $N^{3}$-[2-[3-(4'-bromobiphenyl-4-ylamino)propylamino]ethyl] propane-1,3-diamine] (4d) was obtained as the second product in the synthesis of compound 3d. Eluent $\mathrm{CH}_{2} \mathrm{Cl}_{2}-\mathrm{MeOH}-\mathrm{NH}_{3}$ aq 100:20:2, yield $86 \mathrm{mg}$ (18\%), pale-yellow crystals, m.p. 164$165^{\circ} \mathrm{C} .{ }^{1} \mathrm{H}$ NMR $\delta 1.79$ (quintet, $\left.{ }^{3} \mathrm{~J}=5.8 \mathrm{~Hz}, 8 \mathrm{H}\right), 2.73(\mathrm{~s}, 8 \mathrm{H}), 2.75\left(\mathrm{t},{ }^{3} \mathrm{~J}=6.4 \mathrm{~Hz}, 8 \mathrm{H}\right), 3.20(\mathrm{t}$, $\left.{ }^{3} J=5.9 \mathrm{~Hz}, 8 \mathrm{H}\right), 6.61\left(\mathrm{~d},{ }^{3} \mathrm{~J}=8.2 \mathrm{~Hz}, 4 \mathrm{H}\right), 6.64\left(\mathrm{~d},{ }^{3} J=8.7 \mathrm{~Hz}, 4 \mathrm{H}\right), 7.32-7.39(\mathrm{~m}, 12 \mathrm{H}), 7.46$ $\left(\mathrm{d},{ }^{3} J=8.3 \mathrm{~Hz}, 4 \mathrm{H}\right), \mathrm{NH}$ protons were not assigned; ${ }^{13} \mathrm{C}$ NMR $\delta 29.3(2 \mathrm{C}), 29.5(2 \mathrm{C}), 42.6(2 \mathrm{C})$, 42.8 (2C), 48.1 (4C), 49.4 (4C), 112.8 (4C), 113.0 (4C), 119.7 (2C), 126.9 (4C), 127.6 (8C), 128.2 (2C), 130.3 (2C), 131.5 (4C), 140.1 (2C), 146.9 (2C), 148.2 (2C); HRMS MALDI-TOF $\mathrm{m} / \mathrm{z}$ : calcd. for $\mathrm{C}_{52} \mathrm{H}_{65} \mathrm{Br}_{2} \mathrm{~N}_{8} 959.3699[\mathrm{M}+\mathrm{H}]^{+}$, found 959.3743 .

$N^{1}$-(2-(3-Aminopropylamino)ethyl)- $\boldsymbol{N}^{3}$-(4'-bromobiphenyl-4-yl)propane-1,3-diamine (5d) was obtained as the third product in the synthesis of compound 3d. Eluent $\mathrm{CH}_{2} \mathrm{Cl}_{2}-\mathrm{MeOH}-\mathrm{NH}_{3} \mathrm{aq}$ 10:4:1, yield $131 \mathrm{mg}$ (32\%), pale-yellow oil. ${ }^{1} \mathrm{H}$ NMR $\delta 1.62$ (quintet, ${ }^{3} J=6.7 \mathrm{~Hz}, 2 \mathrm{H}$ ), 1.80 (quintet, $\left.{ }^{3} J=6.4 \mathrm{~Hz}, 2 \mathrm{H}\right), 2.67\left(\mathrm{t},{ }^{3} J=6.6 \mathrm{~Hz}, 2 \mathrm{H}\right), 2.72(\mathrm{~s}, 4 \mathrm{H}), 2.72-2.77(\mathrm{~m}, 4 \mathrm{H}), 3.21\left(\mathrm{t},{ }^{3} J=\right.$ $6.5 \mathrm{~Hz}, 2 \mathrm{H}), 6.63\left(\mathrm{~d},{ }^{3} J=8.2 \mathrm{~Hz}, 2 \mathrm{H}\right), 7.36\left(\mathrm{~d},{ }^{3} \mathrm{~J}=8.2 \mathrm{~Hz}, 2 \mathrm{H}\right), 7.37\left(\mathrm{~d},{ }^{3} J=8.1 \mathrm{~Hz}, 2 \mathrm{H}\right), 7.46$ $\left({ }^{3} \mathrm{~J}=8.1 \mathrm{~Hz}, 2 \mathrm{H}\right), \mathrm{NH}$ protons were not assigned; ${ }^{13} \mathrm{C}$ NMR $\delta 29.1,32.8,40.2,42.4,47.6,47.8$, 49.1, 49.2, 112.7 (2C), 119.6, 127.4 (4C), 128.1, 131.4 (2C), 140.0, 148.1; HRMS MALDI-TOF $\mathrm{m} / \mathrm{z}$ : calcd. for $\mathrm{C}_{20} \mathrm{H}_{30} \mathrm{BrN}_{4} 405.1654[\mathrm{M}+\mathrm{H}]^{+}$, found 405.1670 .

$N, N^{\prime}$-[2,2'-[Ethane-1,2-diylbis(oxy)]bis(ethane-2,1-diyl)]bis(3'-bromobiphenyl-3-amine) (10b) was synthesized from 3,3-dibromobiphenyl $9(0.75 \mathrm{mmol}, 234 \mathrm{mg})$ and dioxadiamine $2 \mathbf{b}(0.25$ $\mathrm{mmol}, 37 \mathrm{mg})$ in the presence of $\mathrm{Pd}(\mathrm{dba})_{2}(6 \mathrm{mg})$ and Xanthphos $(6.5 \mathrm{mg})$ in dioxane $(2.5 \mathrm{ml})$, after $46 \mathrm{~h}$ reflux. Eluent $\mathrm{CH}_{2} \mathrm{Cl}_{2}-\mathrm{MeOH}$ 500:1-200:1, yield $41 \mathrm{mg}$ (27\%), pale-yellow glassy solid. ${ }^{1} \mathrm{H}$ NMR $\delta 3.36\left(\mathrm{t},{ }^{3} \mathrm{~J}=5.2 \mathrm{~Hz}, 4 \mathrm{H}\right), 3.68(\mathrm{~s}, 4 \mathrm{H}), 3.74(\mathrm{t}, 5.2 \mathrm{~Hz}, 4 \mathrm{H}), 4.19$ (br s, 2H), 6.62 $\left(\mathrm{dd},{ }^{3} \mathrm{~J}=8.0 \mathrm{~Hz},{ }^{4} \mathrm{~J}=2.2 \mathrm{~Hz}, 2 \mathrm{H}\right), 6.77\left(\mathrm{t},{ }^{4} \mathrm{~J}=1.6 \mathrm{~Hz}, 2 \mathrm{H}\right), 6.88\left(\mathrm{~d},{ }^{3} \mathrm{~J}=7.6 \mathrm{~Hz}, 2 \mathrm{H}\right), 7.22\left(\mathrm{t},{ }^{3} \mathrm{~J}\right.$ $=7.8 \mathrm{~Hz}, 2 \mathrm{H}), 7.25\left(\mathrm{t},{ }^{3} J=7.7 \mathrm{~Hz}, 2 \mathrm{H}\right), 7.41-7.46(\mathrm{~m}, 4 \mathrm{H}), 7.69\left(\mathrm{t},{ }^{4} \mathrm{~J}=1.6 \mathrm{~Hz}, 2 \mathrm{H}\right) ;{ }^{13} \mathrm{C} \mathrm{NMR} \delta$ $43.5(2 \mathrm{C}), 69.6(2 \mathrm{C}), 70.2(2 \mathrm{C}), 111.6(2 \mathrm{C}), 112.6(2 \mathrm{C}), 116.6(2 \mathrm{C}), 122.7(2 \mathrm{C}), 125.7(2 \mathrm{C})$, 129.7 (2C), 129.9 (2C), 130.0 (2C), 130.1 (2C), 140.8 (2C), 143.8 (2C), 148.6 (2C); HRMS MALDI-TOF $\mathrm{m} / \mathrm{z}$ : calcd. for $\mathrm{C}_{30} \mathrm{H}_{31} \mathrm{Br}_{2} \mathrm{~N}_{2} \mathrm{O}_{2} 609.0752[\mathrm{M}+\mathrm{H}]^{+}$, found 609.0739 .

$N^{3}, N^{3}$-Bis[2-[2-[2-(3'-bromobiphenyl-3-ylamino)ethoxy]ethoxy]ethyl]biphenyl-3,3'-diamine (14b) was obtained as the second product in the synthesis of compound 10b. Eluent $\mathrm{CH}_{2} \mathrm{Cl}_{2}$ MeOH 100:1, yield $6 \mathrm{mg}(5 \%)$, pale-yellow glassy solid. ${ }^{1} \mathrm{H}$ NMR $\delta 3.34\left(\mathrm{t},{ }^{3} J=5.0 \mathrm{~Hz}, 4 \mathrm{H}\right)$, $3.35\left(\mathrm{t},{ }^{3} \mathrm{~J}=5.0 \mathrm{~Hz}, 4 \mathrm{H}\right), 3.66(\mathrm{~s}, 8 \mathrm{H}), 3.72\left(\mathrm{t},{ }^{3} \mathrm{~J}=5.0 \mathrm{~Hz}, 4 \mathrm{H}\right), 3.73\left(\mathrm{t},{ }^{3} \mathrm{~J}=5.0 \mathrm{~Hz}, 4 \mathrm{H}\right), 6.58$ $\left(\mathrm{dd},{ }^{3} J=8.0 \mathrm{~Hz},{ }^{4} J=1.8 \mathrm{~Hz}, 2 \mathrm{H}\right), 6.61\left(\mathrm{dd},{ }^{3} \mathrm{~J}=8.2 \mathrm{~Hz},{ }^{4} \mathrm{~J}=1.5 \mathrm{~Hz}, 2 \mathrm{H}\right), 6.76$ (br s, 2H), 6.79 (br s, 2H), $6.87\left(\mathrm{~d},{ }^{3} J=7.2 \mathrm{~Hz}, 2 \mathrm{H}\right), 6.89\left(\mathrm{~d},{ }^{3} J=7.7 \mathrm{~Hz}, 2 \mathrm{H}\right), 7.13-7.29(\mathrm{~m}, 6 \mathrm{H}), 7.40-7.46(\mathrm{~m}$, $4 \mathrm{H}), 7.68\left(\mathrm{t},{ }^{4} \mathrm{~J}=1.8 \mathrm{~Hz}, 2 \mathrm{H}\right), \mathrm{NH}$ protons were not assigned; ${ }^{13} \mathrm{C}$ NMR $\delta 43.5(4 \mathrm{C}), 69.6(4 \mathrm{C})$, 
70.3 (4C), 111.7 (2C), 112.0 (4C), 112.7 (2C), 116.6 (2C), 116.8 (2C), 122.7 (2C), 125.8 (2C), 129.4 (2C), 129.7 (2C), 130.0 (2C), 130.1 (2C), 130.2 (2C), 140.8 (2C), 142.9 (2C), 143.9 (2C), 148.4 (2C), 148.6 (2C); HRMS MALDI-TOF m/z: calcd. for $\mathrm{C}_{48} \mathrm{H}_{53} \mathrm{Br}_{2} \mathrm{~N}_{4} \mathrm{O}_{4} 907.2434[\mathrm{M}+\mathrm{H}]^{+}$, found 907.2479 .

$N, N^{\prime}-[3,3$ '-[2,2'-Oxybis(ethane-2,1-diyl)bis(oxy)]bis(propane-3,1-diyl)]bis(3'-bromo

biphenyl-3-amine) (10c) was synthesized from 3,3-dibromobiphenyl 9 (0.75 mmol, $234 \mathrm{mg}$ ) and trioxadiamine $2 \mathrm{c}(0.25 \mathrm{mmol}, 55 \mathrm{mg})$ in the presence of $\mathrm{Pd}(\mathrm{dba})_{2}(6 \mathrm{mg})$ and Xanthphos $(6.5$ $\mathrm{mg}$ ) in dioxane $(2.5 \mathrm{ml})$. Eluent $\mathrm{CH}_{2} \mathrm{Cl}_{2}-\mathrm{MeOH} 200: 1$, yield $59 \mathrm{mg}$ (35\%), pale-yellow glassy solid. ${ }^{1} \mathrm{H}$ NMR $\delta 1.90$ (quintet, $\left.{ }^{3} J=6.1 \mathrm{~Hz}, 4 \mathrm{H}\right), 3.28\left(\mathrm{t},{ }^{3} \mathrm{~J}=6.4 \mathrm{~Hz}, 4 \mathrm{H}\right), 3.61\left(\mathrm{t},{ }^{3} \mathrm{~J}=5.8 \mathrm{~Hz}\right.$, $4 \mathrm{H}), 3.62-3.65(\mathrm{~m}, 4 \mathrm{H}), 3.67-3.71(\mathrm{~m}, 4 \mathrm{H}), 4.22($ br s, $2 \mathrm{H}), 6.62\left(\mathrm{dd},{ }^{3} \mathrm{~J}=8.1 \mathrm{~Hz},{ }^{4} \mathrm{~J}=2.3 \mathrm{~Hz}\right.$, 2H), 6.75 (br s, 2H), $6.86\left(\mathrm{~d},{ }^{3} J=7.4 \mathrm{~Hz}, 2 \mathrm{H}\right), 7.23\left(\mathrm{t},{ }^{3} \mathrm{~J}=7.8 \mathrm{~Hz}, 2 \mathrm{H}\right), 7.27\left(\mathrm{t},{ }^{3} J=7.8 \mathrm{~Hz}, 2 \mathrm{H}\right)$, $7.45\left(\mathrm{~d},{ }^{3} \mathrm{~J}=7.9 \mathrm{~Hz}, 2 \mathrm{H}\right) 7.49\left(\mathrm{~d},{ }^{3} \mathrm{~J}=7.8 \mathrm{~Hz}, 2 \mathrm{H}\right), 7.73$ (br s, $\left.2 \mathrm{H}\right) ;{ }^{13} \mathrm{C} \mathrm{NMR} \delta 29.0(2 \mathrm{C}), 41.8$ (2C), 69.7 (2C), 70.2 (2C), $70.6(2 \mathrm{C}), 111.2(2 \mathrm{C}), 112.2(2 \mathrm{C}), 115.9(2 \mathrm{C}), 122.6(2 \mathrm{C}), 125.7$ (2C), 129.6 (2C), 129.9 (2C), 130.0 (2C), 130.1 (2C), 140.7 (2C), 144.0 (2C), 148.9 (2C); HRMS MALDI-TOF m/z: calcd. for $\mathrm{C}_{34} \mathrm{H}_{38} \mathrm{Br}_{2} \mathrm{~N}_{2} \mathrm{O}_{3} 680.1249[\mathrm{M}]^{+}$, found 680.1283 .

$N^{3}, N^{3}$-Bis[3-[2-[2-[3-(3'-bromobiphenyl-3-ylamino)propoxy]ethoxy]ethoxy]propyl]

biphenyl-3,3'-diamine (14c) was obtained as the second product in the synthesis of compound 10c. Eluent $\mathrm{CH}_{2} \mathrm{Cl}_{2}-\mathrm{MeOH} 75: 1-50: 1$, yield $25 \mathrm{mg}$ (19\%), pale-yellow glassy solid. ${ }^{1} \mathrm{H}$ NMR $\delta$ 1.89 (quintet, $\left.{ }^{3} \mathrm{~J}=5.9 \mathrm{~Hz}, 8 \mathrm{H}\right), 3.26\left(\mathrm{t},{ }^{3} \mathrm{~J}=6.3 \mathrm{~Hz}, 4 \mathrm{H}\right), 3.27\left(\mathrm{t},{ }^{3} \mathrm{~J}=6.1 \mathrm{~Hz}, 4 \mathrm{H}\right), 3.56-3.62(\mathrm{~m}$, $16 \mathrm{H}), 3.65-3.69(\mathrm{~m}, 8 \mathrm{H}), 4.16(\mathrm{br} \mathrm{s}, 4 \mathrm{H}), 6.56\left(\mathrm{~d},{ }^{3} \mathrm{~J}=8.2 \mathrm{~Hz}, 2 \mathrm{H}\right), 6.58\left(\mathrm{~d},{ }^{3} \mathrm{~J}=8.3 \mathrm{~Hz}, 2 \mathrm{H}\right)$, 6.74 (br s, 2H), 6.78 (br s, 2H), 6.84 (d, $\left.{ }^{3} \mathrm{~J}=7.5 \mathrm{~Hz}, 2 \mathrm{H}\right), 6.88\left(\mathrm{~d},{ }^{3} \mathrm{~J}=7.2 \mathrm{~Hz}, 2 \mathrm{H}\right), 7.19$ (t, ${ }^{3} \mathrm{~J}=$ $7.8 \mathrm{~Hz}, 2 \mathrm{H}), 7.21\left(\mathrm{t},{ }^{3} \mathrm{~J}=8.2 \mathrm{~Hz}, 2 \mathrm{H}\right), 7.25\left(\mathrm{t},{ }^{3} \mathrm{~J}=8.1 \mathrm{~Hz}, 2 \mathrm{H}\right), 7.44\left(\mathrm{~d},{ }^{3} J=7.1 \mathrm{~Hz}, 2 \mathrm{H}\right), 7.48$ $\left(\mathrm{d},{ }^{3} \mathrm{~J}=6.8 \mathrm{~Hz}, 2 \mathrm{H}\right), 7.71$ (br s, 2H); ${ }^{13} \mathrm{C}$ NMR $\delta 29.0$ (2C), 29.1 (2C), 41.8 (4C), 69.7 (4C), 70.2 (4C), 70.6 (4C), 111.2 (2C), 111.5 (2C), $111.6(2 \mathrm{C}), 112.2$ (2C), $115.9(2 \mathrm{C}), 116.2$ (2C), 122.6 (2C), 125.7 (2C), 129.3 (2C), 129.6 (2C), 129.9 (2C), 130.0 (2C), 130.1 (2C), 140.7 (2C), 143.0 (2C), 144.0 (2C), 148.7 (2C), 149.0 (2C); HRMS MALDI-TOF m/z: calcd. for $\mathrm{C}_{56} \mathrm{H}_{68} \mathrm{Br}_{2} \mathrm{~N}_{4} \mathrm{O}_{6}$ $1050.3506[\mathrm{M}]^{+}$, found 1050.3516 .

$N^{1}, N^{1}$-(Ethane-1,2-diyl)bis[ $\boldsymbol{N}^{\mathbf{3}}$-(3'-bromobiphenyl-3-yl)propane-1,3-diamine] (10d) was synthesized from 3,3-dibromobiphenyl $9(1.1 \mathrm{mmol}, 343 \mathrm{mg})$ and tetraamine 2d $(0.5 \mathrm{mmol}, 87$ $\mathrm{mg})$ in the presence of $\mathrm{Pd}(\mathrm{dba})_{2}(12 \mathrm{mg})$ and BINAP $(14 \mathrm{mg})$ in dioxane $(5 \mathrm{ml})$. Eluent $\mathrm{CH}_{2} \mathrm{Cl}_{2}$ MeOH-NH${ }_{3}$ aq 100:20:1, yield $82 \mathrm{mg}$ (26\%), pale-yellow glassy solid. ${ }^{1} \mathrm{H}$ NMR $\delta 1.81$ (quintet, $\left.{ }^{3} J=6.5 \mathrm{~Hz}, 4 \mathrm{H}\right), 2.76(\mathrm{~s}, 4 \mathrm{H}), 2.78\left(\mathrm{t},{ }^{3} \mathrm{~J}=6.6 \mathrm{~Hz}, 4 \mathrm{H}\right), 3.23\left(\mathrm{t},{ }^{3} \mathrm{~J}=6.5 \mathrm{~Hz}, 4 \mathrm{H}\right), 6.61\left(\mathrm{dd},{ }^{3} J=\right.$ $\left.8.1 \mathrm{~Hz},{ }^{4} \mathrm{~J}=1.5 \mathrm{~Hz}, 2 \mathrm{H}\right), 6.75$ (br s, $\left.2 \mathrm{H}\right), 6.87\left(\mathrm{~d},{ }^{3} \mathrm{~J}=7.6 \mathrm{~Hz}, 2 \mathrm{H}\right), 7.23\left(\mathrm{t},{ }^{3} \mathrm{~J}=7.8 \mathrm{~Hz}, 2 \mathrm{H}\right), 7.27$ $\left(\mathrm{t},{ }^{3} J=7.8 \mathrm{~Hz}, 2 \mathrm{H}\right), 7.45$ (d, $\left.{ }^{3} J=8.1 \mathrm{~Hz}, 2 \mathrm{H}\right), 7.49$ (d, $\left.{ }^{3} J=7.7 \mathrm{~Hz}, 2 \mathrm{H}\right), 7.72$ (br s, 2H), NH protons were not assigned; ${ }^{13} \mathrm{C}$ NMR $\delta 29.4$ (2C), 42.7 (2C), 48.1 (2C), 49.3 (2C), 111.2 (2C), 112.2 (2C), 116.0 (2C), 122.6 (2C), 125.7 (2C), 129.6 (2C), 129.9 (2C), 130.0 (4C), 140.7 (2C), 143.9 (2C), 148.9 (2C); HRMS MALDI-TOF m/z: calcd. for $\mathrm{C}_{32} \mathrm{H}_{37} \mathrm{Br}_{2} \mathrm{~N}_{4} 635.1385[\mathrm{M}+\mathrm{H}]^{+}$, found 635.1370 .

$N^{1}, N^{1}$-(Biphenyl-3,3'-diyl)bis[ $N^{3}$-[2-[3-(3'-bromobiphenyl-3-ylamino)propylamino]ethyl]

propane-1,3-diamine] (14d) was obtained as the second product in the synthesis of compound 
10d. Eluent $\mathrm{CH}_{2} \mathrm{Cl}_{2}-\mathrm{MeOH}-\mathrm{NH}_{3}$ aq 100:20:2, yield $68 \mathrm{mg}(28 \%)$, pale-yellow glassy solid. ${ }^{1} \mathrm{H}$ NMR $\delta 1.79$ (quintet, $\left.{ }^{3} J=5.8 \mathrm{~Hz}, 8 \mathrm{H}\right), 2.74(\mathrm{~s}, 8 \mathrm{H}), 2.75\left(\mathrm{t},{ }^{3} \mathrm{~J}=6.4 \mathrm{~Hz}, 8 \mathrm{H}\right), 3.21\left(\mathrm{t},{ }^{3} J=5.7\right.$ $\mathrm{Hz}, 8 \mathrm{H}), 6.57$ (d, $\left.{ }^{3} J=7.8 \mathrm{~Hz}, 2 \mathrm{H}\right), 6.59$ (d, $\left.{ }^{3} \mathrm{~J}=8.2 \mathrm{~Hz}, 2 \mathrm{H}\right), 6.73$ (br s, 2H), 6.79 (br s, 2H), 6.85 $\left(\mathrm{d},{ }^{3} \mathrm{~J}=7.2 \mathrm{~Hz}, 2 \mathrm{H}\right), 6.89\left(\mathrm{~d},{ }^{3} \mathrm{~J}=6.8 \mathrm{~Hz}, 2 \mathrm{H}\right), 7.17-7.29(\mathrm{~m}, 6 \mathrm{H}), 7.43\left(\mathrm{~d},{ }^{3} \mathrm{~J}=6.9 \mathrm{~Hz}, 2 \mathrm{H}\right), 7.47$ $\left({ }^{3} \mathrm{~J}=7.7 \mathrm{~Hz}, 2 \mathrm{H}\right), 7.71$ (br s, 2H), NH protons were not assigned; ${ }^{13} \mathrm{C}$ NMR $\delta 29.4(2 \mathrm{C}), 29.5$ (2C), 42.7 (4C), 48.1 (4C), 49.4 (4C), 111.5 (2C), 111.6 (4C), 112.2 (2C), 116.0 (2C), 116.3 (2C), 122.6 (2C), 125.7 (2C), 129.3 (2C), 129.6 (2C), 129.9 (2C), 130.1 (4C), 140.7 (2C), 142.9 (2C), 143.9 (2C), 148.7 (2C), 148.9 (2C); HRMS MALDI-TOF m/z: calcd. for $\mathrm{C}_{52} \mathrm{H}_{65} \mathrm{Br}_{2} \mathrm{~N}_{8}$ $959.3699[\mathrm{M}+\mathrm{H}]^{+}$, found 959.3662 .

$N^{3}, N^{3}$-(3,3'-[Ethane-1,2-diylbis(azanediyl))bis(propane-3,1-diyl)]bis[ $N^{3}$-[3-[2-[3-(3'bromobiphenyl-3-ylamino)propylamino]ethylamino]propyl]biphenyl-3,3'-diamine]

obtained as the third product in the synthesis of compound 10d. Eluent $\mathrm{CH}_{2} \mathrm{Cl}_{2}-\mathrm{MeOH}-\mathrm{NH}_{3} \mathrm{aq}$ 100:20:3, yield $12 \mathrm{mg}(12 \%)$, pale-yellow glassy solid. ${ }^{1} \mathrm{H}$ NMR $\delta 1.77$ (br s, 12H), 2.72 (br s, 24H), 3.20 (br s, 12H), 6.55 (d, $\left.{ }^{3} J=7.6 \mathrm{~Hz}, 4 \mathrm{H}\right), 6.57\left(\mathrm{~d},{ }^{3} \mathrm{~J}=8.0 \mathrm{~Hz}, 2 \mathrm{H}\right), 6.72$ (br s, 2H), 6.77 (br s, 4H), $6.84\left(\mathrm{~d},{ }^{3} J=8.5 \mathrm{~Hz}, 2 \mathrm{H}\right), 6.87\left(\mathrm{~d},{ }^{3} \mathrm{~J}=7.0 \mathrm{~Hz}, 4 \mathrm{H}\right), 7.14-7.28(\mathrm{~m}, 8 \mathrm{H}), 7.42\left(\mathrm{~d},{ }^{3} \mathrm{~J}=\right.$ $8.0 \mathrm{~Hz}, 2 \mathrm{H}), 7.46\left(\mathrm{~d},{ }^{3} \mathrm{~J}=7.7 \mathrm{~Hz}, 2 \mathrm{H}\right), 7.69$ (br s, 2H), NH protons were not assigned; ${ }^{13} \mathrm{C}$ NMR

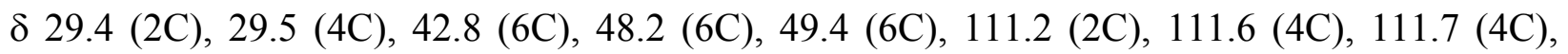
112.3 (2C), 116.0 (2C), 116.3 (4C), 125.8 (2C), 129.4 (4C), 129.7 (2C), 129.9 (2C), 130.0 (2C), 130.1 (4C), 140.8 (2C), 143.0 (4C), 144.0 (2C), 148.7 (4C), 149.0 (2C) HRMS MALDI-TOF $\mathrm{m} / \mathrm{z}$ : calcd. for $\mathrm{C}_{72} \mathrm{H}_{93} \mathrm{Br}_{2} \mathrm{~N}_{12} 1283.6013[\mathrm{M}+\mathrm{H}]^{+}$, found 1283.5970 .

Standard method for the synthesis of cyclic dimers 6a-d and 11b-d via isolated $N, N^{\prime}-$ bis(bromobiphenyl)polyamines (3a-d) and (10b-d)

A two-neck flask equipped with a magnetic stirrer, flushed with dry argon was charged with $N, N^{\prime}$-bis(bromobiphenyl)polyamines 3a-d and 10b-d (1 equiv.), $\operatorname{Pd}(\mathrm{dba})_{2}$ (8 mol\%), BINAP (9 molo\%), absolute dioxane (to make $0.02 \mathrm{M}$ conc.), the mixture was stirred for $2 \mathrm{~min}$, then appropriate polyamine 2a-d ( 1 equiv.) was added, followed by $t \mathrm{BuONa}$ ( 3 equiv.). The reaction mixture was refluxed for $30-40 \mathrm{~h}$, cooled to ambient temperature, filtered and evaporated in vacuo. The residue was chromatographed on silica gel using a sequence of eluents: $\mathrm{CH}_{2} \mathrm{Cl}_{2}$, $\mathrm{CH}_{2} \mathrm{Cl}_{2} / \mathrm{MeOH} 200: 1-3: 1, \mathrm{CH}_{2} \mathrm{Cl}_{2} / \mathrm{MeOH} / \mathrm{NH}_{3}$ aq 100:20:1 - 10:4:1.

$6,10,19,23-T e t r a a z a p e n t a c y c l o\left[22 \cdot 2 \cdot 2 \cdot 2^{2,5} \cdot 2^{11,14} \cdot 2^{15,18}\right]$ tetratriaconta-1(26),2,4,11,13,15,17,24, 27,29,31,33-dodecaene (6a) was synthesized from compound 3a (0.3 mmol, $175 \mathrm{mg}), 1,3$ diaminopropane 2a $(0.3 \mathrm{mmol}, 23 \mathrm{mg})$ in the presence of $\mathrm{Pd}(\mathrm{dba})_{2}(14 \mathrm{mg})$, BINAP $(17 \mathrm{mg})$, $t \mathrm{BuONa}(90 \mathrm{mg})$, in $15 \mathrm{ml}$ dioxane. Isolated as a mixture with cyclic tetramer $7 \mathrm{a}(\mathrm{n}=3)$ Eluent $\mathrm{CH}_{2} \mathrm{Cl}_{2} / \mathrm{MeOH} 75: 1$, yield (for the mixture) $8 \mathrm{mg}(6 \%)^{1} \mathrm{H} \mathrm{NMR} \delta 1.97$ (br s, 4H), 3.30 (br s, $8 \mathrm{H}), 6.42\left(\mathrm{~d},{ }^{3} \mathrm{~J}=8.5 \mathrm{~Hz}, 8 \mathrm{H}\right), 7.07\left(\mathrm{~d},{ }^{3} \mathrm{~J}=8.5 \mathrm{~Hz}, 8 \mathrm{H}\right), \mathrm{NH}$ protons were not assigned; ${ }^{13} \mathrm{C}$ NMR $\delta 29.2$ (2C), 42.2 (4C), 113.2 (8C), 127.2 (8C), quaternary carbons were not detected; MS MALDI-TOF m/z: calcd. for $\mathrm{C}_{30} \mathrm{H}_{33} \mathrm{~N}_{4} 449.27[\mathrm{M}+\mathrm{H}]^{+}$, found 449.25.

$6,10,19,23,32,36,45,49-$ Octaazanonacyclo $\left[48 \cdot 2 \cdot 2 \cdot 2^{2,5} \cdot 2^{11,14} \cdot 2^{15,18} \cdot 2^{24,27} \cdot 2^{28,31} \cdot 2^{37,40} \cdot 2^{41,44}\right]-$ octahexaconta-1(52),2,4,11,13,15,17,24,26,28,30,37,39,41,43,50,53,55,57,59,61,63,65,67- 
tetracosaene (7a, $\mathbf{n = 3}$ ) was isolated together with cyclic dimer $6 \mathbf{6 a} .{ }^{1} \mathrm{H}$ NMR $\delta 1.97$ (br s, 8H), 3.30 (br s, 16H), $6.61\left(\mathrm{~d},{ }^{3} \mathrm{~J}=8.4 \mathrm{~Hz}, 16 \mathrm{H}\right), 7.37\left(\mathrm{~d},{ }^{3} \mathrm{~J}=8.4 \mathrm{~Hz}, 16 \mathrm{H}\right), \mathrm{NH}$ protons were not assigned; ${ }^{13} \mathrm{C}$ NMR $\delta 29.2$ (4C), 42.2 (8C), 113.1 (16C), 127.2 (16C), quaternary carbons were not detected; MS MALDI-TOF m/z: calcd. for $\mathrm{C}_{60} \mathrm{H}_{65} \mathrm{~N}_{8} 897.53[\mathrm{M}+\mathrm{H}]^{+}$, found 897.57.

$9,12,27,30$-Tetraoxa-6,15,24,33-tetraazapentacyclo[32.2.2.2.5 $\left.\cdot 2^{16,19} \cdot 2^{20,23}\right]$ tetratetraconta1(36),2,4,16,18,20,22,34,37,39,41,43-dodecaene (6b) was synthesized from compound $3 \mathbf{b}(0.18$ mmol, $110 \mathrm{mg})$, dioxadiamine $2 \mathbf{b}(0.18 \mathrm{mmol}, 27 \mathrm{mg})$ in the presence of $\mathrm{Pd}(\mathrm{dba})_{2}(8 \mathrm{mg})$, BINAP (10 mg), $t \mathrm{BuONa}(52 \mathrm{mg})$, in $9 \mathrm{ml}$ dioxane. Eluent $\mathrm{CH}_{2} \mathrm{Cl}_{2} / \mathrm{MeOH}$ 50:1, yield $10 \mathrm{mg}$ $(9 \%)$.

\section{$10,13,16,33,36,39-H e x a o x a-6,20,29,43-t e t r a a z a p e n t a c y c l o\left[42.2 \cdot 2 \cdot 2^{2,5} \cdot 2^{21,24} \cdot 2^{25,28}\right]-$}

tetrapentaconta-1(46),2,4,21,23,25,27,44,47,49,51,53-dodecaene (6c) was synthesized from compound 3c $(0.3 \mathrm{mmol}, 205 \mathrm{mg})$, trioxadiamine 2c $(0.3 \mathrm{mmol}, 65 \mathrm{mg})$ in the presence of $\mathrm{Pd}(\mathrm{dba})_{2}(14 \mathrm{mg}), \mathrm{BINAP}(17 \mathrm{mg}), t \mathrm{BuONa}(90 \mathrm{mg})$, in $15 \mathrm{ml}$ dioxane. Eluent $\mathrm{CH}_{2} \mathrm{Cl}_{2} / \mathrm{MeOH}$ $50: 1$, yield $44 \mathrm{mg}(20 \%)$.

$\boldsymbol{N}, \boldsymbol{N}^{\prime}$-[Oxybis(ethane-2,1-diyloxypropane-3,1-diyl)]dibiphenyl-4-amine (8c) was obtained as the second product in the synthesis of compound 6c. Eluent $\mathrm{CH}_{2} \mathrm{Cl}_{2} / \mathrm{MeOH} 100: 1$, yield $25 \mathrm{mg}$ (16\%), slightly beige glassy solid. ${ }^{1} \mathrm{H}$ NMR $\delta 1.91$ (quintet, $\left.{ }^{3} J=5.9 \mathrm{~Hz}, 4 \mathrm{H}\right), 3.28\left(\mathrm{t},{ }^{3} \mathrm{~J}=6.5 \mathrm{~Hz}\right.$, $4 \mathrm{H}), 3.59-3.65(\mathrm{~m}, 8 \mathrm{H}), 3.67-3.72(\mathrm{~m}, 4 \mathrm{H}), 4.05$ (br s, 2H), $6.67\left(\mathrm{~d},{ }^{3} \mathrm{~J}=8.6 \mathrm{~Hz}, 4 \mathrm{H}\right), 7.24\left(\mathrm{t},{ }^{3} \mathrm{~J}\right.$ $=7.9 \mathrm{~Hz}, 2 \mathrm{H}), 7.38\left(\mathrm{t},{ }^{3} \mathrm{~J}=7.7 \mathrm{~Hz}, 4 \mathrm{H}\right), 7.43\left(\mathrm{~d},{ }^{3} \mathrm{~J}=8.5 \mathrm{~Hz}, 4 \mathrm{H}\right), 7.54\left(\mathrm{~d},{ }^{3} J=7.8 \mathrm{~Hz}, 4 \mathrm{H}\right) ;{ }^{13} \mathrm{C}$ NMR $\delta 29.1$ (2C), 41.7 (2C), 69.7 (2C), 70.2 (2C), 70.6 (2C), 112.9 (4C), 125.9 (2C), 126.2 (4C), 127.8 (4C), 128.6 (4C), 129.8 (2C), 141.3 (2C), 148.0 (2C); HRMS MALDI-TOF m/z: calcd. for $\mathrm{C}_{34} \mathrm{H}_{41} \mathrm{~N}_{2} \mathrm{O}_{3} 525.3117[\mathrm{M}+\mathrm{H}]^{+}$, found 525.3092 .

Cyclic oligomers 7c, $(\mathbf{n}=3,5)$ were isolated as a mixture in the synthesis of compound $\mathbf{6 c}$. Eluent $\mathrm{CH}_{2} \mathrm{Cl}_{2} / \mathrm{MeOH}$ 20:1-10:1, yield $50 \mathrm{mg}$ (23\%). ${ }^{1} \mathrm{H}$ NMR $\delta 1.88$ (quintet, ${ }^{3} \mathrm{~J}=5.8 \mathrm{~Hz}$, $4(\mathrm{n}+1) \mathrm{H}), 3.28$ (br s, 4(n+1)H), 3.56-3.64 (m, 8(n+1)H), 3.68 (br s, 4(n+1)H), $6.62\left(\mathrm{~d},{ }^{3} J=8.1\right.$ $\mathrm{Hz}, 4(\mathrm{n}+1) \mathrm{H}), 7.34\left(\mathrm{~d},{ }^{3} J=8.1 \mathrm{~Hz}, 4(\mathrm{n}+1) \mathrm{H}\right)$, NH protons were not assigned; ${ }^{13} \mathrm{C}$ NMR $\delta 29.1$ $(2(n+1) C), 41.9(2(n+1) C), 69.7(2(n+1) C), 70.2(2(n+1) C), 70.6(2(n+1) C), 113.0(4(n+1) C)$, $127.0(4(n+1) C), 128.6(2(n+1) C), 147.0(2(n+1) C) ;$ MS MALDI-TOF $m / z$ : calcd. for $\mathrm{C}_{88} \mathrm{H}_{121} \mathrm{~N}_{8} \mathrm{O}_{12} 1481.91[\mathrm{M}+\mathrm{H}]^{+}$, found 1481.85 (7c, $\mathrm{n}=3$ ); $\mathrm{m} / \mathrm{z}$ : calcd. for $\mathrm{C}_{132} \mathrm{H}_{181} \mathrm{~N}_{12} \mathrm{O}_{18} 2222.36$ $[\mathrm{M}+\mathrm{H}]^{+}$, found $2222.21(7 \mathrm{c}, \mathrm{n}=5)$.

$6,10,13,17,26,30,33,37-O c t a a z a p e n t a c y c l o\left[36 \cdot 2 \cdot 2 \cdot 2^{2,5} \cdot 2^{18,21} \cdot 2^{22,25}\right]$ octatetraconta-1(40),2,4,18, $\mathbf{2 0 , 2 2 , 2 4 , 3 8 , 4 1 , 4 3 , 4 5 , 4 7 - d o d e c a e n e ~ ( 6 d ) ~ w a s ~ s y n t h e s i z e d ~ f r o m ~ c o m p o u n d ~ 3 d ~ ( 0 . 2 6 ~ m m o l , ~} 175$ $\mathrm{mg})$, tetraamine $2 \mathbf{d}(0.26 \mathrm{mmol}, 45 \mathrm{mg})$ in the presence of $\mathrm{Pd}(\mathrm{dba})_{2}(12 \mathrm{mg})$, BINAP (14 $\left.\mathrm{mg}\right)$, $t \mathrm{BuONa}(75 \mathrm{mg})$, in $13 \mathrm{ml}$ dioxane. Eluent $\mathrm{CH}_{2} \mathrm{Cl}_{2} / \mathrm{MeOH} / \mathrm{NH}_{3}$ aq 100:20:2-100:20:3, yield 39 mg (23\%), pale-yellow crystals, m.p. $99-100^{\circ} \mathrm{C} .{ }^{1} \mathrm{H}$ NMR $\delta 1.78$ (quintet, ${ }^{3} J=6.4 \mathrm{~Hz}, 8 \mathrm{H}$ ), 2.75 $(\mathrm{s}, 8 \mathrm{H}), 2.76\left(\mathrm{t},{ }^{3} \mathrm{~J}=6.2 \mathrm{~Hz}, 8 \mathrm{H}\right), 3.18\left(\mathrm{t},{ }^{3} \mathrm{~J}=6.4 \mathrm{~Hz}, 8 \mathrm{H}\right), 6.55\left(\mathrm{~d},{ }^{3} \mathrm{~J}=8.6 \mathrm{~Hz}, 8 \mathrm{H}\right), 7.24\left(\mathrm{~d},{ }^{3} \mathrm{~J}=\right.$ $8.6 \mathrm{~Hz}, 8 \mathrm{H}), \mathrm{NH}$ protons were not assigned; ${ }^{13} \mathrm{C}$ NMR $\delta 29.4(4 \mathrm{C}), 43.1$ (4C), 48.1 (4C), 49.0 (4C), 113.1 (8C), 127.0 (8C), 130.4 (4C), 147.0 (4C); HRMS MALDI-TOF m/z: calcd. for $\mathrm{C}_{40} \mathrm{H}_{57} \mathrm{~N}_{8} 649.4706[\mathrm{M}+\mathrm{H}]^{+}$, found 649.4725. 
Synthesis of cyclic dimer (6c) via in situ obtained compound (3c). A two-neck flask equipped with a magnetic stirrer, flushed with dry argon was charged with 4,4'-dibromobiphenyl 1 (1.25 mmol, $390 \mathrm{mg}), \mathrm{Pd}(\mathrm{dba})_{2}(12 \mathrm{mg})$, BINAP (14 mg), absolute dioxane $(5 \mathrm{ml})$, the mixture was stirred for $2 \mathrm{~min}$, then trioxadiamine $2 \mathrm{c}(0.5 \mathrm{mmol}, 110 \mathrm{mg})$ was added, followed by $t \mathrm{BuONa}$ (144 mg). The reaction mixture was refluxed for $10 \mathrm{~h}$, cooled to ambient temperature, then $\mathrm{Pd}(\mathrm{dba})_{2}(35 \mathrm{mg})$, BINAP (42 $\left.\mathrm{mg}\right)$, absolute dioxane $(20 \mathrm{ml})$ were added, the reaction mixture was stirred for $2 \mathrm{~min}$, trioxadiamine 2c $(0.75 \mathrm{mmol}, 155 \mathrm{mg})$ was added, followed by $t \mathrm{BuONa}$ (200 mg). The reaction mixture was refluxed for $35 \mathrm{~h}$, cooled to ambient temperature, filtered and evaporated in vacuo. The residue was chromatographed on silica gel using a sequence of eluents: $\mathrm{CH}_{2} \mathrm{Cl}_{2}, \mathrm{CH}_{2} \mathrm{Cl}_{2} / \mathrm{MeOH}$ 200:1 - 3:1. Compound 8c was isolated with eluent $\mathrm{CH}_{2} \mathrm{Cl}_{2} / \mathrm{MeOH}$ 75:1 (74 mg, 28\%), cyclic dimer 6c was isolated with eluent $\mathrm{CH}_{2} \mathrm{Cl}_{2} / \mathrm{MeOH}$ 50:1 $(17 \mathrm{mg}, 5 \%)$, a mixture of cyclic dimer $6 \mathrm{c}$ and cyclic trimer $7 \mathrm{c}(\mathrm{n}=2)$ was isolated with eluent $\mathrm{CH}_{2} \mathrm{Cl}_{2} / \mathrm{MeOH}$ 20:1 (34 mg, 9\%).

10,13,16,33,36,39,56,59,62-Nonaoxa-6,20,29,43,52,66-hexaazaheptacyclo $2^{25,28} \cdot 2^{44,47} \cdot 2^{48,51}$ ]henoctaconta-1(69),2,4,21,23,25,27,44,46,48,50,67,70,72,

$\left[65.2 \cdot 2 \cdot 2^{2,5} \cdot 2^{21,24}\right.$ octadecaene (7c, n=2): ${ }^{1} \mathrm{H}$ NMR $\delta 1.88$ (br s, 12H), 2.35 (br s, 12H), 3.58-3.65 (m, 24H), 3.68 (br s, $12 \mathrm{H}), 6.62\left(\mathrm{~d},{ }^{3} J=8.3 \mathrm{~Hz}, 12 \mathrm{H}\right), 7.34\left(\mathrm{~d},{ }^{3} \mathrm{~J}=8.3 \mathrm{~Hz}, 12 \mathrm{H}\right)$, NH protons were not assigned; ${ }^{13} \mathrm{C}$ NMR $\delta 29.1$ (6C), 41.8 (6C), 69.7 (6C), 70.2 (6C), 70.6 (6C), 113.0 (12C), 126.9 (12C), 129.0 (6C), 147.0 (6C); MS MALDI-TOF m/z: calcd. for $\mathrm{C}_{66} \mathrm{H}_{91} \mathrm{~N}_{6} \mathrm{O}_{9} 1111.68[\mathrm{M}+\mathrm{H}]^{+}$, found 1111.73 .

$10,13,30,33$-Tetraoxa-7,16,27,36-tetraazapentacyclo[35.3.1.1 $\cdot^{2,6} \cdot 1^{17,21} \cdot 1^{22,26}$-tetratetraconta1(41),2(44),3,5,17(43),18,20,22(42),23,25,37,39-dodecaene (11b) was synthesized from compound 10b $(0.1 \mathrm{mmol}, 60 \mathrm{mg})$, dioxadiamine $2 \mathbf{b}(0.1 \mathrm{mmol}, 15 \mathrm{mg})$ in the presence of $\mathrm{Pd}(\mathrm{dba})_{2}(4.5 \mathrm{mg})$, BINAP $(5.5 \mathrm{mg}), t \mathrm{BuONa}(29 \mathrm{mg})$, in $5 \mathrm{ml}$ dioxane. Eluent $\mathrm{CH}_{2} \mathrm{Cl}_{2} / \mathrm{MeOH}$ 100:1-75:1, yield $16 \mathrm{mg}(27 \%)$.

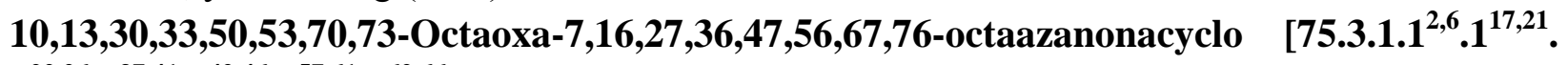
$\left.1^{22,26} .1^{37,41} .1^{42,46} \cdot 1^{57,61} \cdot 1^{62,66}\right]$ octaoctaconta-1(81),2(88),3,5,17(87),18,20, 22(86),23,25,37(85),

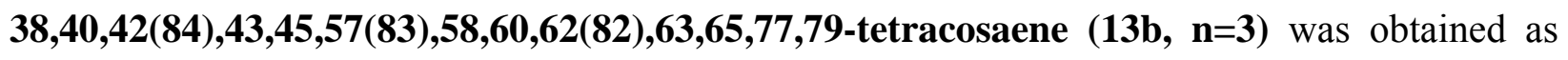
the second product in the synthesis of compound 10b. Eluent $\mathrm{CH}_{2} \mathrm{Cl}_{2} / \mathrm{MeOH} 50: 1$, yield $8 \mathrm{mg}$ (13\%), pale-yellow glassy solid. ${ }^{1} \mathrm{H}$ NMR $\delta 3.31\left(\mathrm{t},{ }^{3} \mathrm{~J}=5.2 \mathrm{~Hz}, 16 \mathrm{H}\right), 3.62(\mathrm{~s}, 16 \mathrm{H}), 3.68\left(\mathrm{t},{ }^{3} \mathrm{~J}=\right.$ $5.2 \mathrm{~Hz}, 16 \mathrm{H}), 6.56\left(\mathrm{~d},{ }^{3} \mathrm{~J}=7.5 \mathrm{~Hz}, 8 \mathrm{H}\right), 6.79(\mathrm{~s}, 8 \mathrm{H}), 6.88\left(\mathrm{~d},{ }^{3} J=7.3 \mathrm{~Hz}, 8 \mathrm{H}\right), 7.15\left(\mathrm{t},{ }^{3} J=7.8\right.$ $\mathrm{Hz}, 8 \mathrm{H})$, NH protons were not assigned; ${ }^{13} \mathrm{C}$ NMR $\delta 43.5(8 \mathrm{C}), 69.6(8 \mathrm{C}), 70.2(8 \mathrm{C}), 111.9(8 \mathrm{C})$, 112.1 (8C), 116.7 (8C), 129.4 (8C), 142.8 (8C), 148.4 (8C), HRMS MALDI-TOF m/z: calcd. for $\mathrm{C}_{72} \mathrm{H}_{89} \mathrm{~N}_{8} \mathrm{O}_{8} 1193.6803[\mathrm{M}+\mathrm{H}]^{+}$, found 1193.6758.

Cyclic oligomers (13b, $\mathbf{n}=\mathbf{3}, \mathbf{5})$ were isolated as a mixture in a separate fraction in the synthesis of cyclic dimer 11b. Eluent $\mathrm{CH}_{2} \mathrm{Cl}_{2} / \mathrm{MeOH} 20: 1$, yield $11 \mathrm{mg}$ (18\%). Cyclic hexamer 13b $(\mathrm{n}=5):{ }^{1} \mathrm{H}$ NMR $\delta 3.31$ (br s, 24H), 3.63 (s, 24H), 3.68 (br s, 24H), 3.98 (br s, 12H), 6.57 (d, ${ }^{3} J=$ $8.1 \mathrm{~Hz}, 12 \mathrm{H}), 6.78(\mathrm{~s}, 12 \mathrm{H}), 6.88\left(\mathrm{~d},{ }^{3} \mathrm{~J}=7.3 \mathrm{~Hz}, 12 \mathrm{H}\right), 7.16\left(\mathrm{t},{ }^{3} \mathrm{~J}=7.6 \mathrm{~Hz}, 12 \mathrm{H}\right) ;{ }^{13} \mathrm{C} \mathrm{NMR} \delta$ 43.5 (12C), 69.7 (12C), 70.2 (12C), 111.9 (12C), 112.0 (12C), 116.8 (12C), 129.4 (12C), 142.8 
(12C), 148.4 (12C); MS MALDI-TOF m/z: calcd. for $\mathrm{C}_{108} \mathrm{H}_{133} \mathrm{~N}_{12} \mathrm{O}_{12} 1790.02[\mathrm{M}+\mathrm{H}]^{+}$, found 1790.14 .

\section{1,14,17,36,39,42-Hexaoxa-7,21,32,46-tetraazapentacyclo[45.3.1.1 $\left.1^{2,6} \cdot 1^{22,26} \cdot 1^{27,31}\right]$-tetrapentaconta-} 1(51),2(54),3,5,22(53),23,25,27(52),28,30,47,49-dodecaene (11c) was synthesized from compound 10c $(0.085 \mathrm{mmol}, 58 \mathrm{mg})$, trioxadiamine 2c $(0.085 \mathrm{mmol}, 19 \mathrm{mg})$ in the presence of $\mathrm{Pd}(\mathrm{dba})_{2}(4 \mathrm{mg})$, BINAP (5 mg), tBuONa (26 mg), in $4 \mathrm{ml}$ dioxane. Eluent $\mathrm{CH}_{2} \mathrm{Cl}_{2} / \mathrm{MeOH} 75: 1-$ 50:1, yield $19 \mathrm{mg}(30 \%)$.

Cyclic oligomers 13c $(\mathbf{n}=3, \mathbf{5})$ were isolated as a mixture in a separate fraction in the synthesis of cyclic dimer 11c, contains also cyclic dimer 11c. Eluent $\mathrm{CH}_{2} \mathrm{Cl}_{2} / \mathrm{MeOH}$ 50:1-10:1, yield 30 mg (48\%). ${ }^{1} \mathrm{H}$ NMR $\delta 1.87$ (quintet, $\left.{ }^{3} J=5.5 \mathrm{~Hz}, 4(\mathrm{n}+1) \mathrm{H}\right), 3.23\left(\mathrm{t},{ }^{3} J=6.0 \mathrm{~Hz}, 4(\mathrm{n}+1) \mathrm{H}\right), 3.52-$ $3.60(\mathrm{~m}, 8(\mathrm{n}+1) \mathrm{H}), 3.61-3.66(\mathrm{~m}, 4(\mathrm{n}+1) \mathrm{H}), 6.55\left(\mathrm{~d},{ }^{3} J=6.6 \mathrm{~Hz}, 2(\mathrm{n}+1) \mathrm{H}\right), 6.76($ br s, 2(n+1)H), $6.86\left(\mathrm{~d},{ }^{3} J=7.5 \mathrm{~Hz}, 2(\mathrm{n}+1) \mathrm{H}\right), 7.17\left(\mathrm{t},{ }^{3} J=7.3 \mathrm{~Hz}, 2(\mathrm{n}+1) \mathrm{H}\right), \mathrm{NH}$ protons were not assigned; ${ }^{13} \mathrm{C}$ NMR $\delta 29.1(2(n+1) C), 41.8(2(n+1) C), 69.7(2(n+1) C), 70.2(2(n+1) C), 70.6(2(n+1) C), 111.6$ $(4(n+1) C), 116.2(2(n+1) C), 129.3(2(n+1) C), 142.9(2(n+1) C), 148.7(2(n+1) C)$; MS MALDITOF m/z: calcd. for $\mathrm{C}_{88} \mathrm{H}_{121} \mathrm{~N}_{8} \mathrm{O}_{12} 1481.91[\mathrm{M}+\mathrm{H}]^{+}$, found $1481.85(13, \mathrm{n}=3) ; \mathrm{m} / \mathrm{z}$ : calcd. for $\mathrm{C}_{132} \mathrm{H}_{181} \mathrm{~N}_{12} \mathrm{O}_{18} 2222.36[\mathrm{M}+\mathrm{H}]^{+}$, found $2222.24(13, \mathrm{n}=5)$.

$7,11,14,18,29,33,36,40-O c t a a z a p e n t a c y c l o\left[39.3 .1 .1^{2,6} \cdot 1^{19,23} \cdot 1^{24,28}\right]$ octatetraconta-1(45),2(48), 3,5,19(47),20,22,24(46),25,27,41,43-dodecaene (11d) was synthesized from compound 10d $(0.19 \mathrm{mmol}, 120 \mathrm{mg})$, tetraamine $2 \mathrm{~d}(0.19 \mathrm{mmol}, 33 \mathrm{mg})$ in the presence of $\mathrm{Pd}(\mathrm{dba})_{2}(9 \mathrm{mg})$, BINAP $(11 \mathrm{mg}), t \mathrm{BuONa}(55 \mathrm{mg})$, in $10 \mathrm{ml}$ dioxane. Eluent $\mathrm{CH}_{2} \mathrm{Cl}_{2} / \mathrm{MeOH} / \mathrm{NH}_{3} \mathrm{aq}$ 100:20:3, yield $31 \mathrm{mg}(25 \%)$.

7,11,14,18,29,33,36,40,51,55,58,62,73,77,80,84-Hexadecaazanonacyclo $\left[83 \cdot 3 \cdot 1.1^{2,6} \cdot 1^{19,23} \cdot 1^{24,28}\right.$. $1^{41,45} .1^{46,50} .1^{63,67} .1^{68,72}$ ]hexanonaconta-1(89),2(96),3,5,19(95),20,22,24(94),25,27,41(93),42,44, 46(92),47,49,63(91),64,66,68(90),69,71,85,87-tetracosaene $(13 d, n=3)$ was obtained as a mixture with cyclic compound 11d in a separate fraction. Eluent $\mathrm{CH}_{2} \mathrm{Cl}_{2} / \mathrm{MeOH} / \mathrm{NH}_{3} \mathrm{aq}$ 100:25:5, yield (for a mixture) $34 \mathrm{mg}(28 \%) .{ }^{1} \mathrm{H}$ NMR $\delta 1.75$ (br s, $\left.16 \mathrm{H}\right), 2.70$ (br s, 32H), 3.16 (br s, 16H), 6.53 (br s, 8H), 6.76 (br s, 8H), 6.86 (br s, 8H), 7.15 (br s, 8H), NH protons were not assigned; ${ }^{13} \mathrm{C}$ NMR $\delta 29.4(8 \mathrm{C}), 42.7$ (8C), 48.1 (8C), 49.2 (8C), $111.5(8 \mathrm{C}), 111.6(8 \mathrm{C}), 116.2$ (8C), 129.4 (8C), 142.9 (8C), 148.8 (8C); MS MALDI-TOF m/z: calcd. for $\mathrm{C}_{80} \mathrm{H}_{113} \mathrm{~N}_{16} 1297.93$ $[\mathrm{M}+\mathrm{H}]^{+}$, found 1297.84 .

Standard method for the synthesis of cyclic dimers 11a,c,d via in situ obtained $N, N^{\prime}-$ bis(bromobiphenyl)polyamines (10a,c,d)

A two-neck flask equipped with a magnetic stirrer, flushed with dry argon was charged with 3,3'dibromobiphenyl 9 (1.25 mmol, $390 \mathrm{mg}$ ), Pd(dba) 2 (12 mg), BINAP (14 mg), absolute dioxane $(5 \mathrm{ml})$, the mixture was stirred for $2 \mathrm{~min}$, then appropriate polyamine 2a,c,d $(0.5 \mathrm{mmol})$ was added, followed by $t \mathrm{BuONa}(144 \mathrm{mg})$. The reaction mixture was refluxed for $10 \mathrm{~h}$, cooled to ambient temperature, then $\mathrm{Pd}(\mathrm{dba})_{2}(34 \mathrm{mg})$, BINAP (41 mg), absolute dioxane (20 ml) were added, the reaction mixture was stirred for $2 \mathrm{~min}$, appropriate polyamine 2a,c,d $(0.6 \mathrm{mmol})$ was added, followed by $t \mathrm{BuONa}(170 \mathrm{mg})$. The reaction mixture was refluxed for $30-40 \mathrm{~h}$, cooled to 
ambient temperature, filtered and evaporated in vacuo. The residue was chromatographed on silica gel using a sequence of eluents: $\mathrm{CH}_{2} \mathrm{Cl}_{2}, \mathrm{CH}_{2} \mathrm{Cl}_{2} / \mathrm{MeOH}$ 200:1 $\quad$ - 3:1, $\mathrm{CH}_{2} \mathrm{Cl}_{2} / \mathrm{MeOH} / \mathrm{NH}_{3}$ aq 100:20:1 - 10:4:1.

7,11,22,26-Tetraazapentacyclo[25.3.1.1 $1^{2,6} .1^{12,16} .1^{17,21}$ ]tetratriaconta-1(31),2(34),3,5,12(33),13,15, 17(32),18,20,27,29-dodecaene (11a) was synthesized from 1,3-diaminopropane 2a. Eluent $\mathrm{CH}_{2} \mathrm{Cl}_{2} / \mathrm{MeOH} 200: 1$, yield $12 \mathrm{mg}$ (5\%).

$7,11,22,26,37,41-H e x a a z a h e p t a c y c l o\left[40.3 .1 .1^{2,6} \cdot 1^{12,16} \cdot 1^{17,21} \cdot 1^{27,31} \cdot 1^{32,36}\right]$ henpentaconta-1(46), 2(51),3,5,12(50),13,15,17(49),18,20,27(48),28,30,32(47),33,35,42,44-octadecaene $(13 a, \quad n=2)$ was obtained as the second product in the synthesis of cyclic dimer 11a and isolated as a mixture with it in a separate fraction. Eluent $\mathrm{CH}_{2} \mathrm{Cl}_{2} / \mathrm{MeOH} 100: 1$, yield (for a mixture) $35 \mathrm{mg}(15 \%) .{ }^{1} \mathrm{H}$ NMR $\delta 1.93$ (quintet, $\left.{ }^{3} J=6.3 \mathrm{~Hz}, 6 \mathrm{H}\right), 3.29\left(\mathrm{t},{ }^{3} \mathrm{~J}=6.3 \mathrm{~Hz}, 12 \mathrm{H}\right), 3.63(\mathrm{br} \mathrm{s}, 6 \mathrm{H}), 6.58\left(\mathrm{~d},{ }^{3} \mathrm{~J}=\right.$ $7.6 \mathrm{~Hz}, 6 \mathrm{H}), 6.76$ (br s, 6H), $6.90\left(\mathrm{~d},{ }^{3} \mathrm{~J}=7.7 \mathrm{~Hz}, 6 \mathrm{H}\right), 7.20\left(\mathrm{t},{ }^{3} J=7.8 \mathrm{~Hz}, 6 \mathrm{H}\right) ;{ }^{13} \mathrm{C}$ NMR $\delta 29.1$ (3C), 41.9 (6C), 111.7 (6C), 111.8 (6C), 116.6 (6C), 129.5 (6C), 142.8 (6C), 148.4 (6C); MS MALDI-TOF m/z: calcd. for $\mathrm{C}_{45} \mathrm{H}_{49} \mathrm{~N}_{6} 673.40[\mathrm{M}+\mathrm{H}]^{+}$, found 673.42 .

Cyclic dimer (11b) was synthesized from dioxadiamine 2b. Eluent $\mathrm{CH}_{2} \mathrm{Cl}_{2} / \mathrm{MeOH}$ 75:1, yield $24 \mathrm{mg}(8 \%)$.

10,13-Dioxa-7,16-diazatricyclo[15.3.1.1 $\left.{ }^{2,6}\right]$ docosa-1(21),2(22),3,5,17,19-hexaene (12b) was obtained as the second product in the synthesis of cyclic dimer 11b Eluent $\mathrm{CH}_{2} \mathrm{Cl}_{2} / \mathrm{MeOH} 100: 1$, yield $15 \mathrm{mg}(10 \%)$.

Cyclic oligomers (13b, $\mathbf{n}=\mathbf{2}, \mathbf{3})$ was obtained as a separate mixture in the synthesis of cyclic dimer 11b. Eluent $\mathrm{CH}_{2} \mathrm{Cl}_{2} / \mathrm{MeOH}$ 50:1-20:1, yield $20 \mathrm{mg}$ (7\%).

Cyclic dimer (11d) was synthesized from tetraamine 2d. Eluent $\mathrm{CH}_{2} \mathrm{Cl}_{2} / \mathrm{MeOH} / \mathrm{NH}_{3} \mathrm{aq}$ 100:25:5, yield $63 \mathrm{mg}(19 \%)$.

7,11,14,18-Tetraazatricyclo[17.3.1.1 $\left.{ }^{2,6}\right]$ tetracosa-1(23),2(24),3,5,19,21-hexaene (12d) was obtained as the second product in the synthesis of cyclic dimer 11d Eluent $\mathrm{CH}_{2} \mathrm{Cl}_{2} / \mathrm{MeOH} / \mathrm{NH}_{3} \mathrm{aq}$ 100:20:1-100:20:2, yield $30 \mathrm{mg}$ (18\%).

7,11,14,18,29,33,36,40,51,55,58,62-Dodecaazaheptacyclo-[61.3.1.1 $1^{2,6} \cdot 1^{19,23} \cdot 1^{24,28} \cdot 1^{41,45} \cdot 1^{46,50}$ ] doheptaconta-1(67),2(72),3,5,19(71),20,22,24(70),25,27,41(69),42,44,46(68),47,49,63,65octadecaene (13d, $\mathbf{n = 2}$ ) was obtained as a mixture with cyclic dimer $\mathbf{1 1 d}$ in a separate fraction. Eluent $\mathrm{CH}_{2} \mathrm{Cl}_{2} / \mathrm{MeOH} / \mathrm{NH}_{3}$ aq 100:35:8, yield (for a mixture) $38 \mathrm{mg}(12 \%)$. ${ }^{1} \mathrm{H} \mathrm{NMR} \delta 1.78$ (br s, $12 \mathrm{H}), 2.70(\mathrm{br} \mathrm{s}, 24 \mathrm{H}), 3.18\left(\mathrm{t},{ }^{3} \mathrm{~J}=7.0 \mathrm{~Hz}, 12 \mathrm{H}\right), 6.55(\mathrm{br} \mathrm{s}, 6 \mathrm{H}), 6.77(\mathrm{br} \mathrm{s}, 6 \mathrm{H}), 6.87\left(\mathrm{~d},{ }^{3} \mathrm{~J}=\right.$ $6.2 \mathrm{~Hz}, 6 \mathrm{H}), 7.16\left(\mathrm{t},{ }^{3} \mathrm{~J}=6.9 \mathrm{~Hz}, 6 \mathrm{H}\right)$, NH protons were not assigned; ${ }^{13} \mathrm{C}$ NMR $\delta 29.4(6 \mathrm{C}), 42.6$ (6C), 48.0 (6C), 49.2 (6C), 111.5 (12C), 116.2 (6C), 129.3 (6C), 142.8 (6C), 148.7 (6C); MS MALDI-TOF $\mathrm{m} / \mathrm{z}$ : calcd. for $\mathrm{C}_{60} \mathrm{H}_{85} \mathrm{~N}_{12} 973.70[\mathrm{M}+\mathrm{H}]^{+}$, found 973.74 .

Standard method for the synthesis of cyclic dimers 6b-d and $11 \mathrm{~b}-\mathrm{d}$ via in situ obtained bis(polyamine) substituted biphenyls (16b-d) and (18b-d)

A two-neck flask equipped with a magnetic stirrer, flushed with dry argon was charged with 4,4'dibromobiphenyl 1 or 3,3'-dibromobiphenyl 9 (0.25 mmol, $78 \mathrm{mg}), \operatorname{Pd}(\mathrm{dba})_{2}(6 \mathrm{mg})$, BINAP (7 $\mathrm{mg})$, absolute dioxane $(2.5 \mathrm{ml})$, the mixture was stirred for $2 \mathrm{~min}$, then appropriate polyamine 
2b-d ( $1 \mathrm{mmol})$ was added, followed by $t \mathrm{BuONa}(72 \mathrm{mg})$. The reaction mixture was refluxed for $10 \mathrm{~h}$, cooled to ambient temperature, $0.25 \mathrm{ml}$ of the reaction mixture was taken for NMR and mass-spectroscopic investigations of compounds $\mathbf{1 6}$ and 18, then $\mathrm{Pd}(\mathrm{dba})_{2}$ (28 $\left.\mathrm{mg}\right)$, BINAP (34 $\mathrm{mg})$, absolute dioxane $(10 \mathrm{ml})$ were added, the reaction mixture was stirred for $2 \mathrm{~min}, 4,4^{\prime}-$ dibromobiphenyl 1 or 3,3'-dibromobiphenyl $9(0.6 \mathrm{mmol}, 187 \mathrm{mg})$ was added, followed by $t \mathrm{BuONa}(170 \mathrm{mg})$. The reaction mixture was refluxed for $30-40 \mathrm{~h}$, cooled to ambient temperature, filtered and evaporated in vacuo. The residue was chromatographed on silica gel using a sequence of eluents: $\mathrm{CH}_{2} \mathrm{Cl}_{2}, \mathrm{CH}_{2} \mathrm{Cl}_{2} / \mathrm{MeOH}$ 200:1 - 3:1, $\mathrm{CH}_{2} \mathrm{Cl}_{2} / \mathrm{MeOH} / \mathrm{NH}_{3}$ aq 100:20:1 - 10:4:1.

$\boldsymbol{N}, \boldsymbol{N}^{\prime}$-Bis[2-[2-(2-aminoethoxy)ethoxy]ethyl]biphenyl-4,4'-diamine (16b) was obtained in situ from dioxadiamine $2 \mathbf{b}(1 \mathrm{mmol}, 148 \mathrm{mg}) .{ }^{1} \mathrm{H}$ NMR $\delta 2.82\left(\mathrm{t},{ }^{3} \mathrm{~J}=5.1 \mathrm{~Hz}, 4 \mathrm{H}\right), 3.29\left(\mathrm{t},{ }^{3} \mathrm{~J}=5.2\right.$ $\mathrm{Hz}, 4 \mathrm{H}), 3.48$ (t, $\left.{ }^{3} J=5.2 \mathrm{~Hz}, 4 \mathrm{H}\right), 3.59-3.63(\mathrm{~m}, 8 \mathrm{H}), 3.69\left(\mathrm{t},{ }^{3} \mathrm{~J}=5.2 \mathrm{~Hz}, 4 \mathrm{H}\right), 6.64\left(\mathrm{~d},{ }^{3} J=8.6\right.$ $\mathrm{Hz}, 4 \mathrm{H}), 7.34\left(\mathrm{~d},{ }^{3} \mathrm{~J}=8.6 \mathrm{~Hz}, 4 \mathrm{H}\right)$, NH protons were not assigned; ${ }^{13} \mathrm{C}$ NMR $\delta 41.5(2 \mathrm{C}), 43.5$ (2C), 69.5 (2C), 70.2 (4C), 73.4 (2C), 113.4 (4C), 127.0 (4C), 130.8 (2C), 146.7 (2C); MS MALDI-TOF m/z: calcd. for $\mathrm{C}_{24} \mathrm{H}_{39} \mathrm{~N}_{4} \mathrm{O}_{4} 447.30[\mathrm{M}+\mathrm{H}]^{+}$, found 447.32.

Cyclic dimer 6b was synthesized from compound 16b. Eluent $\mathrm{CH}_{2} \mathrm{Cl}_{2} / \mathrm{MeOH}$ 75:1, yield $16 \mathrm{mg}$ $(11 \%)$.

$9,12,27,30,45,48-H e x a o x a-6,15,24,33,42,51-h e x a a z a h e p t a c y c l o-\left[50.2 .2 .2^{2,5} \cdot 2^{16,19} \cdot 2^{20,23} 2^{34,37} \cdot 2^{38,41}\right]$

hexahexaconta-1(54),2,4,16,18,20,22,34,36,38,40,52,55,57,59,61,63,65-octadecaene $(7 \mathbf{b}, \mathbf{n = 2})$ was obtained as the second product in the synthesis of cyclic dimer $6 \mathbf{b} . \mathrm{CH}_{2} \mathrm{Cl}_{2} / \mathrm{MeOH} 75: 1$, yield $14 \mathrm{mg}(9 \%)$.

A mixture of cyclic oligomers $(\mathbf{7 b}, \mathbf{n}=\mathbf{2 - 4})$ was obtained as a separate fraction in the synthesis of cyclic dimer $\mathbf{6 b}$. Eluent $\mathrm{CH}_{2} \mathrm{Cl}_{2} / \mathrm{MeOH} 50: 1$, yield $17 \mathrm{mg}$ (11\%).

$9,12,27,30,45,48,63,66-O c t a o x a-6,15,24,33,42,51,60,69-o c t a a z a n o n a c y c l o-\left[68.2 .2 .2^{2,5} \cdot 2^{16,19}\right.$. $2^{20,23} \cdot 2^{34,37} \cdot 2^{38,41} \cdot 2^{52,55} \cdot 2^{56,59}$ ]octaoctaconta-1(72),2,4,16,18,20,22,34,36,38,

40,52,54,56,58,70,73,75,77,79,81,83,85,87-tetracosaene (7b, $\mathbf{n}=3)$. ${ }^{1} \mathrm{H}$ NMR $\delta 3.31\left(\mathrm{t},{ }^{3} J=5.0\right.$ $\mathrm{Hz}, 16 \mathrm{H}), 3.64(\mathrm{~s}, 16 \mathrm{H}), 3.72\left(\mathrm{t},{ }^{3} J=5.0 \mathrm{~Hz}, 16 \mathrm{H}\right), 6.59\left(\mathrm{~d},{ }^{3} J=8.6 \mathrm{~Hz}, 16 \mathrm{H}\right), 7.27\left(\mathrm{~d},{ }^{3} J=8.6\right.$ $\mathrm{Hz}, 16 \mathrm{H}), \mathrm{NH}$ protons were not assigned; ${ }^{13} \mathrm{C}$ NMR $\delta 43.7(8 \mathrm{C}), 69.6(8 \mathrm{C}), 70.3(8 \mathrm{C}), 113.5$ (16C), 127.1 (16C), 130.8 (8C), 146.6 (8C); MS MALDI-TOF m/z: calcd. for $\mathrm{C}_{72} \mathrm{H}_{89} \mathrm{~N}_{8} \mathrm{O}_{8}$ $1193.68[\mathrm{M}+\mathrm{H}]^{+}$, found 1193.62 .

9,12,27,30,45,48,63,66,81,84-Decaoxa-6,15,24,33,42,51,60,69,78,87-decaazaundecacyclo $\left[86 \cdot 2 \cdot 2 \cdot 2^{2,5} \cdot 2^{16 \cdot 19} \cdot 2^{20,23} \cdot 2^{34,37} \cdot 2^{38,41} \cdot 2^{52,55} \cdot 2^{56,59} \cdot 2^{70,73} \cdot 2^{74,77}\right]$ decahecta-1(90),2,4,16,18,20,22,34,36,

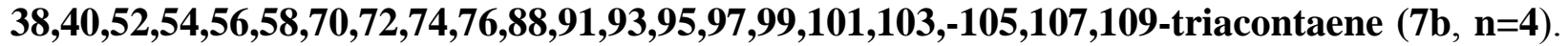
${ }^{1} \mathrm{H}$ NMR $\delta 3.31\left(\mathrm{t},{ }^{3} J=5.0 \mathrm{~Hz}, 20 \mathrm{H}\right), 3.64(\mathrm{~s}, 20 \mathrm{H}), 372\left(\mathrm{t},{ }^{3} J=5.0 \mathrm{~Hz}, 20 \mathrm{H}\right), 6.62\left(\mathrm{~d},{ }^{3} J=8.5\right.$ $\mathrm{Hz}, 20 \mathrm{H}), 7.32\left(\mathrm{~d},{ }^{3} \mathrm{~J}=8.5 \mathrm{~Hz}, 20 \mathrm{H}\right)$, NH protons were not assigned; ${ }^{13} \mathrm{C}$ NMR $\delta 43.7(10 \mathrm{C})$, 69.7 (10C), 70.3 (10C), 113.5 (20C), 127.1 (20C), 130.8 (10C), 146.7 (10C); MS MALDI-TOF $\mathrm{m} / \mathrm{z}$ : calcd. for $\mathrm{C}_{90} \mathrm{H}_{111} \mathrm{~N}_{10} \mathrm{O}_{10} 1491.85[\mathrm{M}+\mathrm{H}]^{+}$, found 1491.78.

Cyclic oligomers (7b, $\mathbf{n = 3 - 8 )}$ were obtained as a separate fraction in the synthesis of cyclic dimer 6b. Eluent $\mathrm{CH}_{2} \mathrm{Cl}_{2} / \mathrm{MeOH} 20: 1$, yield $43 \mathrm{mg}(29 \%)$. 7b, $\mathrm{n}>4:{ }^{1} \mathrm{H}$ NMR $\delta 3.33$ (br s, $4(n+1) H), 3.65(s, 4(n+1) H), 3.71\left(\right.$ br s, 4(n+1)H), $3.99($ br s, $2(n+1) H), 6.65\left(d,{ }^{3} J=8.2 \mathrm{~Hz}\right.$, 
$4(\mathrm{n}+1) \mathrm{H}), 7.35\left(\mathrm{~d},{ }^{3} \mathrm{~J}=8.2 \mathrm{~Hz}, 4(\mathrm{n}+1) \mathrm{H}\right) ;{ }^{13} \mathrm{C}$ NMR $\delta 43.6(2(\mathrm{n}+1) \mathrm{C}), 69.6(2(\mathrm{n}+1) \mathrm{C}), 70.2$ $(2(n+1) C), 113.4(4(n+1) C), 127.0(4(n+1) C), 130.7(2(n+1) C), 146.7(2(n+1) C)$; MS MALDITOF m/z: calcd. for $\mathrm{C}_{108} \mathrm{H}_{133} \mathrm{~N}_{12} \mathrm{O}_{12} 1790.0[\mathrm{M}+\mathrm{H}]^{+}$, found $1789.97 \mathbf{b}(\mathrm{n}=5) ; \mathrm{m} / \mathrm{z}$ : calcd. for $\mathrm{C}_{126} \mathrm{H}_{155} \mathrm{~N}_{14} \mathrm{O}_{14} 2088.2[\mathrm{M}+\mathrm{H}]^{+}$, found $2088.0 \mathbf{7 b}(\mathrm{n}=6) ; \mathrm{m} / \mathrm{z}$ : calcd. for $\mathrm{C}_{144} \mathrm{H}_{177} \mathrm{~N}_{16} \mathrm{O}_{16} 2386.4$ $[\mathrm{M}+\mathrm{H}]^{+}$, found $2386.1(7 \mathbf{b}, \mathrm{n}=7) ; \mathrm{m} / \mathrm{z}$ : calcd. for $\mathrm{C}_{162} \mathrm{H}_{199} \mathrm{~N}_{18} \mathrm{O}_{18} 2684.5[\mathrm{M}+\mathrm{H}]^{+}$, found 2684.1 $(7 \mathbf{b}, \mathrm{n}=8)$.

$N^{4}, N^{4}$-Bis(3-(2-(2-(3-aminopropoxy)ethoxy)ethoxy)propyl)biphenyl-4,4'-diamine (16c) was obtained in situ from trioxadiamine 2c (1mmol, $220 \mathrm{mg}) .{ }^{1} \mathrm{H}$ NMR $\delta 1.67$ (quintet, ${ }^{3} \mathrm{~J}=6.5 \mathrm{~Hz}$, 4H), 1.85 (quintet, $\left.{ }^{3} \mathrm{~J}=5.2 \mathrm{~Hz}, 4 \mathrm{H}\right), 2.71\left(\mathrm{t},{ }^{3} \mathrm{~J}=6.7 \mathrm{~Hz}, 4 \mathrm{H}\right), 3.20\left(\mathrm{t},{ }^{3} \mathrm{~J}=6.4 \mathrm{~Hz}, 4 \mathrm{H}\right), 3.50\left(\mathrm{t},{ }^{3} \mathrm{~J}\right.$ $=6.2 \mathrm{~Hz}, 4 \mathrm{H}), 3.52-3.64(\mathrm{~m}, 20 \mathrm{H}), 6.59\left(\mathrm{~d},{ }^{3} \mathrm{~J}=8.6 \mathrm{~Hz}, 4 \mathrm{H}\right), 7.30\left(\mathrm{~d},{ }^{3} \mathrm{~J}=8.6 \mathrm{~Hz}, 4 \mathrm{H}\right), \mathrm{NH}$ protons were not assigned; ${ }^{13} \mathrm{C}$ NMR $\delta 29.0$ (2C), 33.2 (2C), 39.3 (2C), 41.7 (2C), 69.3 (2C), 69.6 (2C), 70.0 (2C), 70.1 (2C), 70.5 (4C), 112.9 (4C), 126.9 (4C), 130.3 (2C), 146.9 (2C); MS MALDI-TOF m/z: calcd. for $\mathrm{C}_{32} \mathrm{H}_{55} \mathrm{~N}_{4} \mathrm{O}_{6} 591.41[\mathrm{M}+\mathrm{H}]^{+}$, found 591.38.

Cyclic dimer (6c) was synthesized from compound 16c. Eluent $\mathrm{CH}_{2} \mathrm{Cl}_{2} / \mathrm{MeOH}$ 50:1-20:1, yield $46 \mathrm{mg}(24 \%)$.

Cyclic trimer (7c, $\mathbf{n = 2}$ ) was obtained as the second product in the synthesis of cyclic dimer $\mathbf{6 c}$. Eluent $\mathrm{CH}_{2} \mathrm{Cl}_{2} / \mathrm{MeOH} 20: 1$, yield $8 \mathrm{mg}$ (4\%).

Oligomers (17c, $\mathbf{n = 1 - 4 )}$ were isolated as separate mixture in the synthesis of cyclic dimer 6c. Eluent $\mathrm{CH}_{2} \mathrm{Cl}_{2} / \mathrm{MeOH}$ 10:1-5:1, yield $94 \mathrm{mg}$ (50\%). 1.81 (br s, $2 \mathrm{H}$ ), 1.88 (quintet, ${ }^{3} \mathrm{~J}=5.0 \mathrm{~Hz}$, $(4 \mathrm{n}+2) \mathrm{H}), 2.99($ br s, $2 \mathrm{H}), 3.23\left(\mathrm{t},{ }^{3} J=5.2 \mathrm{~Hz},(4 \mathrm{n}+2) \mathrm{H}\right), 4.59($ br s, $(2 \mathrm{n}+1) \mathrm{H}), 6.62\left(\mathrm{~d},{ }^{3} J=8.1\right.$ $\mathrm{Hz}, 4 \mathrm{nH}), 6.70\left(\mathrm{~d},{ }^{3} \mathrm{~J}=8.6 \mathrm{~Hz}, 2 \mathrm{H}\right), 7.24\left(\mathrm{t},{ }^{3} \mathrm{~J}=8.0 \mathrm{~Hz}, 1 \mathrm{H}\right), 7.34\left(\mathrm{~d},{ }^{3} J=8.1 \mathrm{~Hz}, 4 \mathrm{nH}\right), 7.38(\mathrm{t}$, $\left.{ }^{3} J=7.7 \mathrm{~Hz}, 2 \mathrm{H}\right), 7.42\left(\mathrm{~d},{ }^{3} \mathrm{~J}=8.5 \mathrm{~Hz}, 2 \mathrm{H}\right), 7.52\left(\mathrm{~d},{ }^{3} \mathrm{~J}=7.5 \mathrm{~Hz}, 2 \mathrm{H}\right)$, protons of $\mathrm{NH}_{2}$ group were not assigned; ${ }^{13} \mathrm{C}$ NMR $\delta 26.6$ (1C), 28.8 (1C), 29.0 (2nC), 39.7 (1C), 41.6 (1C), 41.7 (2nC), $69.6(2(\mathrm{n}+1) \mathrm{C}), 70.1(2(\mathrm{n}+1) \mathrm{C}), 70.5(2(\mathrm{n}+1) \mathrm{C}), 112.9(4 \mathrm{nC}), 113.5(2 \mathrm{C}), 126.0(2 \mathrm{C}), 126.9$ $(4 \mathrm{nC}), 127.7(2 \mathrm{C}), 128.5(2 \mathrm{C}), 129.0(1 \mathrm{C}), 130.2(2 \mathrm{nC}), 146.9(2(\mathrm{n}+1) \mathrm{C})$, two aromatic quaternary carbons were not detected; MS MALDI-TOF m/z: calcd. for $\mathrm{C}_{44} \mathrm{H}_{63} \mathrm{~N}_{4} \mathrm{O}_{6} 743.47$ $[\mathrm{M}+\mathrm{H}]^{+}$, found $743.44(\mathbf{1 7 c}, \mathrm{n}=1) ; \mathrm{m} / \mathrm{z}$ : calcd. for $\mathrm{C}_{66} \mathrm{H}_{93} \mathrm{~N}_{6} \mathrm{O}_{9} 1113.70[\mathrm{M}+\mathrm{H}]^{+}$, found 1113.65 17c $(\mathrm{n}=2) ; \mathrm{m} / \mathrm{z}$ : calcd. for $\mathrm{C}_{88} \mathrm{H}_{123} \mathrm{~N}_{8} \mathrm{O}_{12} 1483.93[\mathrm{M}+\mathrm{H}]^{+}$, found 1483.85 17c $(\mathrm{n}=3)$; $\mathrm{m} / \mathrm{z}$ : calcd. for $\mathrm{C}_{110} \mathrm{H}_{153} \mathrm{~N}_{10} \mathrm{O}_{15} 1854.15[\mathrm{M}+\mathrm{H}]^{+}$, found 1854.01 17c $(\mathrm{n}=4)$.

$N^{1}, N^{1}$-(Biphenyl-4,4'-diyl)bis[ $\boldsymbol{N}^{\mathbf{3}}$-[2-(3-aminopropylamino)ethyl]propane-1,3-diamine] (16d) was obtained in situ from tetraamine $2 \mathbf{d}\left(1 \mathrm{mmol}, 174 \mathrm{mg}\right.$ ). ${ }^{1} \mathrm{H}$ NMR $\delta 1.58$ (quintet, ${ }^{3} \mathrm{~J}=6.9 \mathrm{~Hz}$, 4H), 1.76 (quintet, $\left.{ }^{3} J=6.4 \mathrm{~Hz}, 4 \mathrm{H}\right), 2.61\left(\mathrm{t},{ }^{3} \mathrm{~J}=7.0 \mathrm{~Hz}, 4 \mathrm{H}\right), 2.67(\mathrm{~s}, 8 \mathrm{H}), 2.68-2.72(\mathrm{~m}, 8 \mathrm{H})$, $3.16\left(\mathrm{t},{ }^{3} \mathrm{~J}=6.4 \mathrm{~Hz}, 4 \mathrm{H}\right), 6.59\left(\mathrm{~d},{ }^{3} \mathrm{~J}=8.5 \mathrm{~Hz}, 4 \mathrm{H}\right), 7.31\left(\mathrm{~d},{ }^{3} \mathrm{~J}=8.5 \mathrm{~Hz}, 4 \mathrm{H}\right), \mathrm{NH}$ protons were not assigned; ${ }^{13} \mathrm{C}$ NMR $\delta 29.5$ (2C), 33.8 (2C), 40.2 (2C), 42.8 (2C), $47.6(2 \mathrm{C}), 48.0(2 \mathrm{C}), 49.4$ (4C), 112.9 (4C), 129.9 (4C), 130.4 (2C), 146.9 (2C); MS MALDI-TOF m/z: calcd. for $\mathrm{C}_{28} \mathrm{H}_{51} \mathrm{~N}_{8}$ $499.42[\mathrm{M}+\mathrm{H}]^{+}$, found 499.43 .

Cyclic dimer (6d) was synthesized from compound 16d. Eluent $\mathrm{CH}_{2} \mathrm{Cl}_{2} / \mathrm{MeOH} / \mathrm{NH}_{3} \mathrm{aq}$ 100:20:3, yield $12 \mathrm{mg}(7 \%)$.

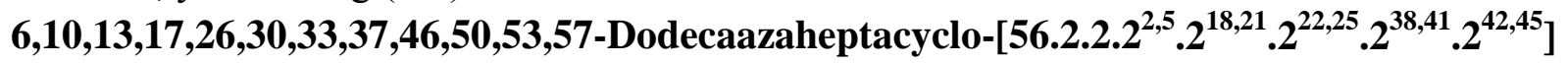
doheptaconta-1(60),2,4,18,20,22,24,38,40,42,44,58,61,63,65,67,69,71-octadecaene $(7 \mathrm{~d}, \mathrm{n}=2)$ 
was obtained as the second product in the synthesis of cyclic dimer 6d. Eluent $\mathrm{CH}_{2} \mathrm{Cl}_{2} / \mathrm{MeOH} / \mathrm{NH}_{3}$ aq 100:20:3, yield $6 \mathrm{mg}$ (4\%), pale-yellow glassy solid. ${ }^{1} \mathrm{H} \mathrm{NMR} \delta 1.77$ (quintet, $\left.{ }^{3} J=6.5 \mathrm{~Hz}, 12 \mathrm{H}\right), 2.73(\mathrm{~s}, 12 \mathrm{H}), 2.76\left(\mathrm{t},{ }^{3} \mathrm{~J}=6.2 \mathrm{~Hz}, 12 \mathrm{H}\right), 3.13\left(\mathrm{t},{ }^{3} \mathrm{~J}=6.4 \mathrm{~Hz}, 12 \mathrm{H}\right)$, $6.60\left(\mathrm{~d},{ }^{3} \mathrm{~J}=8.5 \mathrm{~Hz}, 12 \mathrm{H}\right), 7.33\left(\mathrm{~d},{ }^{3} \mathrm{~J}=8.5 \mathrm{~Hz}, 12 \mathrm{H}\right), \mathrm{NH}$ protons were not assigned; ${ }^{13} \mathrm{C}$ NMR $\delta 29.2$ (6C), 42.9 (6C), 48.1 (6C), 49.0 (6C), 113.1 (12C), 127.0 (12C), 130.4 (6C), 147.0 (6C); HRMS MALDI-TOF m/z: calcd. for $\mathrm{C}_{60} \mathrm{H}_{85} \mathrm{~N}_{12} 973.7020[\mathrm{M}+\mathrm{H}]^{+}$, found 973.7039 .

$N^{3}, N^{3}$-Bis[2-[2-(2-aminoethoxy)ethoxy]ethyl]biphenyl-3,3'-diamine (18b) was obtained in situ from dioxadiamine $2 \mathbf{b}(1 \mathrm{mmol}, 148 \mathrm{mg}) .{ }^{1} \mathrm{H}$ NMR $\delta 2.83\left(\mathrm{t},{ }^{3} \mathrm{~J}=5.2 \mathrm{~Hz}, 4 \mathrm{H}\right), 3.32\left(\mathrm{t},{ }^{3} \mathrm{~J}=\right.$ $5.2 \mathrm{~Hz}, 4 \mathrm{H}), 3.49\left(\mathrm{t},{ }^{3} \mathrm{~J}=5.2 \mathrm{~Hz}, 4 \mathrm{H}\right), 3.61-3.65(\mathrm{~m}, 8 \mathrm{H}), 3.71\left(\mathrm{t},{ }^{3} \mathrm{~J}=5.2 \mathrm{~Hz}, 4 \mathrm{H}\right), 6.58\left(\mathrm{dd},{ }^{3} \mathrm{~J}=\right.$ $\left.8.0 \mathrm{~Hz},{ }^{4} \mathrm{~J}=2.3 \mathrm{~Hz}, 2 \mathrm{H}\right), 6.80\left(\mathrm{t},{ }^{4} \mathrm{~J}=1.9 \mathrm{~Hz}, 2 \mathrm{H}\right), 6.89\left(\mathrm{~d},{ }^{3} \mathrm{~J}=7.6 \mathrm{~Hz}, 2 \mathrm{H}\right), 7.19\left(\mathrm{t},{ }^{3} \mathrm{~J}=7.8 \mathrm{~Hz}\right.$, 2H), NH protons were not assigned; ${ }^{13} \mathrm{C}$ NMR $\delta 41.5$ (2C), 43.4 (2C), $69.6(2 \mathrm{C}), 70.2(4 \mathrm{C}), 73.4$ (2C), 112.0 (4C), 116.7 (2C), 129.3 (2C), 142.8 (2C), 148.4 (2C); MS MALDI-TOF m/z: calcd. for $\mathrm{C}_{24} \mathrm{H}_{39} \mathrm{~N}_{4} \mathrm{O}_{4} 447.30[\mathrm{M}+\mathrm{H}]^{+}$, found 447.27.

Cyclic dimer (11b) was synthesized from compound 18b. Eluent $\mathrm{CH}_{2} \mathrm{Cl}_{2} / \mathrm{MeOH}$ 100:1, yield 66 mg (44\%).

Cyclic oligomers $(\mathbf{1 3 b}, \mathbf{n}=\mathbf{2}, \mathbf{3})$ were obtained as a separate fraction in the synthesis of cyclic dimer 11b. Eluent $\mathrm{CH}_{2} \mathrm{Cl}_{2} / \mathrm{MeOH}$ 50:1, yield $56 \mathrm{mg}$ (32\%).

Cyclic trimer (13b, $\mathbf{n}=\mathbf{2})$ was isolated as a mixture with cyclic dimer $\mathbf{1 1 b}$ in a separate fraction. Eluent $\mathrm{CH}_{2} \mathrm{Cl}_{2} / \mathrm{MeOH} 20: 1$, yield $46 \mathrm{mg}$ (24\%).

Macrocycle (12b) was obtained as the second product in the synthesis of cyclic dimer $\mathbf{1 1 b}$ Eluent $\mathrm{CH}_{2} \mathrm{Cl}_{2} / \mathrm{MeOH} 20: 1$, yield $24 \mathrm{mg}$ (15\%).

$N^{3}, N^{3}$-Bis[3-[2-[2-(3-aminopropoxy)ethoxy]ethoxy]propyl]biphenyl-3,3'-diamine (18c) was obtained in situ from trioxadiamine 2c (1mmol, $220 \mathrm{mg}$ ). ${ }^{1} \mathrm{H}$ NMR $\delta 1.64$ (quintet, ${ }^{3} \mathrm{~J}=6.5 \mathrm{~Hz}$, 4H), 1.85 (quintet, $\left.{ }^{3} \mathrm{~J}=5.6 \mathrm{~Hz}, 4 \mathrm{H}\right), 2.70\left(\mathrm{t},{ }^{3} \mathrm{~J}=6.8 \mathrm{~Hz}, 4 \mathrm{H}\right), 3.21\left(\mathrm{t},{ }^{3} \mathrm{~J}=5.5 \mathrm{~Hz}, 4 \mathrm{H}\right), 3.45-3.60$ $(\mathrm{m}, 24 \mathrm{H}), 6.51\left(\mathrm{~d},{ }^{3} \mathrm{~J}=8.0 \mathrm{~Hz}, 2 \mathrm{H}\right), 6.72(\mathrm{~s}, 2 \mathrm{H}), 6.81\left(\mathrm{~d},{ }^{3} \mathrm{~J}=7.4 \mathrm{~Hz}, 2 \mathrm{H}\right), 7.12\left(\mathrm{t},{ }^{3} \mathrm{~J}=7.7 \mathrm{~Hz}\right.$, 2H), NH protons were not assigned; ${ }^{13} \mathrm{C}$ NMR $\delta 29.0$ (2C), 33.2 (2C), $39.4(2 \mathrm{C}), 41.6(2 \mathrm{C}), 69.3$ (2C), 69.6 (2C), 70.0 (2C), 70.1 (2C), 70.5 (4C), 111.4 (4C), 116.0 (2C), 129.2 (2C), 142.8 (2C), 148.6 (2C); MS MALDI-TOF m/z: calcd. for $\mathrm{C}_{32} \mathrm{H}_{55} \mathrm{~N}_{4} \mathrm{O}_{6} 591.41[\mathrm{M}+\mathrm{H}]^{+}$, found 591.39.

Cyclic dimer (11c) was synthesized from compound 18c. Eluent $\mathrm{CH}_{2} \mathrm{Cl}_{2} / \mathrm{MeOH}$ 75:1-50:1, yield $28 \mathrm{mg}(15 \%)$.

11,14,17-Trioxa-7,21-diazatricyclo[20.3.1.1 ${ }^{2,6}$ ]heptacosa-1(26),2(27),3,5,22,24-hexaene (12c) was obtained as the second product in the synthesis of cyclic dimer 11c. Eluent $\mathrm{CH}_{2} \mathrm{Cl}_{2} / \mathrm{MeOH}$ 100:1, yield $72 \mathrm{mg}(39 \%)$.

Cyclic oligomers (13c, $\mathbf{n = 2 , 3 )}$ were obtained as a separate fraction in the synthesis of cyclic dimer 11c. Eluent $\mathrm{CH}_{2} \mathrm{Cl}_{2} / \mathrm{MeOH} 20: 1$, yield $56 \mathrm{mg}$ (30\%).

$11,14,17,36,39,42,61,64,67-N o n a o x a-7,21,32,46,57,71-h e x a a z a h e p t a c y c l o-\left[70.3 .1 .11^{2,6} \cdot 1^{22,26}\right.$. $1^{27,31} .1^{47,51} .1^{52,56}$ ]henoctaconta-1(76),2(81),3,5,22(80),23,25,27(79),28,30,47(78),48,50,52(77), 53,55,72,74-octadecaene (13c, $\mathbf{n}=2)$. ${ }^{1} \mathrm{H}$ NMR $\delta 1.87$ (quintet, $\left.{ }^{3} \mathrm{~J}=6.0 \mathrm{~Hz}, 12 \mathrm{H}\right), 3.24\left(\mathrm{t},{ }^{3} \mathrm{~J}=\right.$ $6.2 \mathrm{~Hz}, 12 \mathrm{H}), 3.53-3.62(\mathrm{~m}, 24 \mathrm{H}), 3.63-3.68(\mathrm{~m}, 12 \mathrm{H}), 4.00$ (br s, 6H), 6.55 (br s, 6H), $6.78(\mathrm{~s}$, $6 \mathrm{H}), 6.88$ (br s, 6H), $7.18\left(\mathrm{t},{ }^{3} \mathrm{~J}=7,7 \mathrm{~Hz}, 6 \mathrm{H}\right) ;{ }^{13} \mathrm{C} \mathrm{NMR} \delta 29.0(6 \mathrm{C}), 41.7$ (6C), 69.6 (6C), 70.2 
(6C), 70.5 (6C), 111.4 (6C), 111.6 (6C), 116.1 (6C), 129.2 (6C), 142.9 (6C), 148.7 (6C); MS MALDI-TOF m/z: calcd. for $\mathrm{C}_{66} \mathrm{H}_{91} \mathrm{~N}_{6} \mathrm{O}_{9} 1111.68[\mathrm{M}+\mathrm{H}]^{+}$, found 1111.63.

11,14,17,36,39,42,61,64,67,86,89,92-Dodecaoxa-7,21,32,46,57,71,82,96-octaazanonacyclo [95.3.1.1 ${ }^{2,6} \cdot 1^{22,26} \cdot 1^{27,31} \cdot 1^{47,51} \cdot 1^{52,56} \cdot 1^{72,76} \cdot 1^{77,81}$ ]octahecta-1(101),2(108),3,5,22(107),23,25, 27(106),28,30,47(105),48,50,52(104),53,55,72(103),73,75,77(102),78,80,97,99-tetracosaene (13c, $\mathbf{n}=3$ ). ${ }^{1} \mathrm{H}$ NMR $\delta 1.88$ (quintet, $\left.{ }^{3} J=6.2 \mathrm{~Hz}, 16 \mathrm{H}\right), 3.25\left(\mathrm{t},{ }^{3} \mathrm{~J}=6.2 \mathrm{~Hz}, 16 \mathrm{H}\right), 3.53-3.62(\mathrm{~m}$, $32 \mathrm{H}), 3.63-3.68(\mathrm{~m}, 16 \mathrm{H}), 4.05$ (br s, $8 \mathrm{H}), 6.57$ (br s, 8H), $6.78(\mathrm{~s}, 8 \mathrm{H}), 6.88$ (br s, 8H), $7.19(\mathrm{t}$, $\left.{ }^{3} J=7.8 \mathrm{~Hz}, 8 \mathrm{H}\right) ;{ }^{13} \mathrm{C}$ NMR $\delta 29.0(8 \mathrm{C}), 41.7$ (8C), 69.6 (8C), 70.2 (8C), 70.5 (8C), 111.5 (16C), 116.1 (8C), 129.2 (8C), 142.9 (8C), 148.7 (8C); MS MALDI-TOF m/z: calcd. for $\mathrm{C}_{88} \mathrm{H}_{121} \mathrm{~N}_{8} \mathrm{O}_{12}$ $1481.91[\mathrm{M}+\mathrm{H}]^{+}$, found 1481.83 .

$N^{1}, N^{1}$-(Biphenyl-3,3'-diyl)bis[ $N^{3}$-[2-(3-aminopropylamino)ethyl]propane-1,3-diamine] (18d) was obtained in situ from tetraamine $2 \mathbf{d}\left(1 \mathrm{mmol}, 174 \mathrm{mg}\right.$ ). ${ }^{1} \mathrm{H}$ NMR $\delta 1.58$ (quintet, ${ }^{3} \mathrm{~J}=7.0 \mathrm{~Hz}$, 4H), 1.77 (quintet, $\left.{ }^{3} J=5.9 \mathrm{~Hz}, 4 \mathrm{H}\right), 2.62\left(\mathrm{t},{ }^{3} \mathrm{~J}=7.0 \mathrm{~Hz}, 4 \mathrm{H}\right), 2.67(\mathrm{~s}, 8 \mathrm{H}), 2.68-2.73(\mathrm{~m}, 8 \mathrm{H})$, $3.18\left(\mathrm{t},{ }^{3} \mathrm{~J}=5.7 \mathrm{~Hz}, 4 \mathrm{H}\right), 6.53\left(\mathrm{~d},{ }^{3} \mathrm{~J}=7.3 \mathrm{~Hz}, 2 \mathrm{H}\right), 6.74(\mathrm{~s}, 2 \mathrm{H}), 6.83\left(\mathrm{~d},{ }^{3} \mathrm{~J}=7.3 \mathrm{~Hz}, 2 \mathrm{H}\right), 7.15(\mathrm{t}$, $\left.{ }^{3} J=7.6 \mathrm{~Hz}, 2 \mathrm{H}\right)$, NH protons were not assigned; ${ }^{13} \mathrm{C}$ NMR $\delta 29.5$ (2C), $33.8(2 \mathrm{C}), 40.3(2 \mathrm{C})$, 42.7 (2C), 47.7 (2C), 48.1 (2C), 49.5 (4C), 111.4 (2C), 111.5 (2C), 116.2 (2C), 129.3 (2C), 142.9 (2C), 148.7 (2C); MS MALDI-TOF m/z: calcd. for $\mathrm{C}_{28} \mathrm{H}_{51} \mathrm{~N}_{8} 499.42[\mathrm{M}+\mathrm{H}]^{+}$, found 499.40

Cyclic dimer (11d) was synthesized from compound 18d. Eluent $\mathrm{CH}_{2} \mathrm{Cl}_{2} / \mathrm{MeOH} / \mathrm{MH}_{3} \mathrm{aq}$ 100:20:3, yield $14 \mathrm{mg}(9 \%)$.

Macrocycle (12d) was obtained as the second product in the synthesis of cyclic dimer 11d. Eluent $\mathrm{CH}_{2} \mathrm{Cl}_{2} / \mathrm{MeOH} / \mathrm{NH}_{3}$ aq 100:20:1-100:20:2, yield $65 \mathrm{mg}$ (40\%).

\section{Acknowledgements}

This work was supported by RFBR grants N 09-03-00735, 08-03-00628, by the Russian Academy of Sciences program "Elaboration of the methods for the synthesis of chemical compounds and construction of new materials" and by the ARCUS project Bourgogne-Russie.

\section{References}

1. Kohama, H.; Yoshinaga, M.; Ishizu, K. Bull. Chem. Soc. Jpn. 1980, 53, 3707.

2. Reinhoudt, D. N.; de Jong, F.; van de Vondervoort, E. M. Tetrahedron 1981, 37, 1753.

3. Reinhoudt, D. N.; de Jong, F.; van de Vondervoort, E. M. Tetrahedron 1981, 37, 1985.

4. Rebek Jr., J. Acc. Chem. Res. 1984, 17, 258.

5. Rebek Jr., J.; Costello, T.; Marshall, L.; Wattley, R.; Gadwood, R. C.; Onan, K. J. Am. Chem. Soc. 1985, 107, 7481 .

6. Rebek Jr., J.; Wattley, R. V.; Costello, T.; Gadwood, R.; Marshall, L. J. Am. Chem. Soc. 1980, 102, 7398 . 
7. Gaviña, F.; Luis, S. V.; Costero, A. M.; Burguete, M. I.; Rebek, Jr., J. J. Am. Chem. Soc. 1988, 110, 7140.

8. Kotlyar, S. A.; Fonari, M. S.; Simonov, Yu. A.; Bocelli, G.; Shishkin, O. V.; Shishkina, S. V.; Tkachuk, V. V.; Grygorash, R. Ya.; Kamalov, G. L. J. Incl. Phenom. Macrocyclic Chem. 2005, 52, 75.

9. Shishkina, S. V.; Shishkin, O. V.; Grygorash, R. Ya.; Mazepa, A. V.; Rakipov, I. M.; Yakshin, V. V.; Kotlyar, S. A.; Kamalov, G. L. J. Mol. Struct. 2007, 832, 199.

10. Burguete, M. I.; Diaz, P.; Garcia-España, E.; Luis, S. V.; Miravet, J. F.; Querol, M.; Ramirez, J. A. Chem. Commun. 1999, 649.

11. Pearson, D. P. J.; Leigh, S. J.; Sutherland, I. O. J. Chem. Soc., Perkin Trans. 1 1979, 3113.

12. Koschabek, R.; Gleiter, R.; Rominger, F. Eur. J. Inorg. Chem. 2006, 609.

13. Costero, A. M.; Bañuls, M. J.; Aurell, M. J.; de Arellano, M. C. R. J. Incl. Phenom. Macrocyclic Chem. 2006, 54, 61.

14. Costero, A. M.; Bañuls, M. J.; Aurell, M. J.; Ochando, L. E.; Domenech, A. Tetrahedron 2005, 61, 10309.

15. Montero, A.; Albericio, F.; Royo, M.; Herradón, B. Eur. J. Org. Chem. 2007, 1301.

16. Artz, S. P.; Cram, D. J. J. Am. Chem. Soc. 1984, 106, 2160.

17. Choi, K.; Hamilton, A. D. J. Am. Chem. Soc. 2003, 125, 10241.

18. Kuhnert, N.; Straßnig, K.; Lopez-Periago, A. M. Tetrahedron: Asymmetry 2002, 13, 123.

19. Kuhnert, N.; Rossignolo, G. M.; Lopez-Periago, A. M. Org. Biomol. Chem. 2003, 1, 1157.

20. Kuhnert, N.; Patel, C.; Jami, F. Tetrahedron Lett. 2005, 46, 7575.

21. Wei, H.; Zhang, Y. J.; Wang, F.; Zhang, W. Tetrahedron: Asymmetry 2008, 19, 482.

22. Colquhoun, H. M.; Zhu, Zh.; Williams, D. J.; Drew, M. G. B.; Cardin, C. J.; Gan, Y.; Crawford, A. G.; Marder, T. B. Chem. Eur. J. 2010, 16, 907.

23. Colquhoun, H. M.; Greenland, B. W.; Zhu, Zh.; Shaw, J. S.; Cardin, C. J.; Burattini, S.; Elliott, J. M.; Basu, S.; Gasa, T. B.; Stoddart, J. F. Org. Lett. 2009, 11, 5238.

24. Werz, D. B.; Fischer, F. R.; Kornmayer, S. C.; Rominger, F.; Gleiter, R. J. Org. Chem. 2008, $73,8021$.

25. Geng, M.; Zhang, D.; Wu, X.; He, L.; Gong, B. Org. Lett. 2009, 11, 923.

26. Patel, H. K.; Kilburn, J. D.; Langley, G. J.; Edwards, P. D.; Mitchell, T.; Southgate, R. Tetrahedron Lett. 1994, 35, 481.

27. Tominaga, M.; Hatano, T.; Uchiyama, M.; Masu, H.; Kagechika, H.; Azumaya, I. Tetrahedron Lett. 2006, 47, 9369.

28. Miller, A. D.; McBee, J. L.; Tilley, T. D. J. Org. Chem. 2009, 74, 2880.

29. Lloyd-Williams, P.; Giralt, E. Chem. Soc. Rev. 2001, 30, 145.

30. Averin, A. D.; Uglov, A. N.; Buryak, A. K., Beletskaya, I. P. Mendeleev Commun. 2010, 20, 1.

31. Averin, A. D.; Uglov, A. N.; Buryak, A. K., Beletskaya, I. P. Macroheterocycles 2009, 2, 275.

32. Wolfe, J. P.; Buchwald, S. L. J. Org. Chem. 2000, 65, 1147. 
33. Beletskaya, I. P.; Bessmertnykh, A. G.; Averin, A. D.; Denat, F.; Guilard, R. Eur. J. Org. Chem. 2005, 261.

34. Snyder, H. R.; Weaver, C.; Marshall, C. D. J. Am. Chem. Soc. 1949, 71, 289.

35. Ukai, T.; Kawazura, H.; Ishii, Y.; Bonnet, J. J.; Ibers, J. A. J. Organomet. Chem. 1974, 65, 253. 